\title{
$\mathfrak{g l}_{N}$ Higgsed networks
}

\section{Yegor Zenkevich}

SISSA, via Bonomea 265, 34136 Trieste, Italy

INFN, Sezione di Trieste, IGAP, via Beirut 2/1, 34151 Trieste, Italy

ITEP, Bolshaya Cheremushkinskaya street 25, 117218 Moscow, Russia

ITMP MSU, Leninskie gory 1, 119991 Moscow, Russia

MIPT, Institutskii pereulok 9, 141700, Dolgoprudny, Russia

E-mail: yegor.zenkevich@gmail.com

ABstRACT: We generalize the framework of Higgsed networks of intertwiners to the quantum toroidal algebra associated to Lie algebra $\mathfrak{g l}_{N}$. Using our formalism we obtain a systems of screening operators corresponding to $W$-algebras associated to toric strip geometries and reproduce partition functions of $3 d$ theories on orbifolded backgrounds.

KeYwords: Conformal and W Symmetry, Supersymmetric Gauge Theory, Topological Strings

ArXiv ePrint: 1912.13372 


\section{Contents}

1 Introduction 1

2 The intertwiners 3

3 Correlators 4

$3.1 R$-matrix 5

4 Screenings and networks $\quad 6$

4.1 Screening operators 6

$\begin{array}{lll}4.2 & \text { Networks and partition functions } & 7\end{array}$

$\begin{array}{lll}5 & \text { Conclusions } & 9\end{array}$

A The algebra $U_{q_{1}, q_{2}, q_{3}}\left(\widehat{\widehat{\mathfrak{g l}}}_{N}\right) \quad 10$

$\begin{array}{lll}\text { A.1 Coalgebra structure } & 12\end{array}$

$\begin{array}{lll}\text { A.2 Symmetries of the algebra } & 12\end{array}$

A.3 The limit of $U_{q_{1}, q_{2}, q_{3}}\left(\widehat{\mathfrak{\mathfrak { g l }}}_{N}\right)$ to $W_{1+\infty}\left[\mathfrak{g l}_{N}\right]$ and the quantum torus 13

A.4 Vector representations of $U_{q_{1}, q_{2}, q_{3}}\left(\widehat{\hat{\mathfrak{g l}}}_{N}\right) \quad 14$

A.5 Horizontal Fock representation of $U_{q_{1}, q_{2}, q_{3}}\left(\widehat{\hat{\mathfrak{g}}}_{N}\right) \quad 16$

A.6 Branching into $U_{q_{1}, q_{2}, q_{3}}\left(\widehat{\hat{\mathfrak{g l}}}_{1}\right)$ algebras and toric geometry 18

$\begin{array}{ll}\text { B Intertwining property of the higgsed vertex } & 20\end{array}$

\section{Introduction}

Quantum toroidal algebras $[1,2]$ are quantum deformations of double loop algebras. They are natural from several points of view and have intricate representation theory. Most importantly for us, they play a crucial role in different versions of the AGT correspondence [3$5]$, which in its abstract form [6,7] is a statement about actions of certain algebras on the spaces of BPS objects in gauge theories. In many cases the algebras featuring in the correspondence are quantum toroidal algebras and the spaces of BPS states are $K$-theories of various moduli spaces. Here we will not be concerned with the mechanism of the action itself, which belongs to the geometric representation theory (see e.g. [8]). We will use the representation theory of the algebras to build certain intertwining operators and screening currents and with their help compute partition functions of $3 d$ gauge theories. In doing so we follow closely our previous work [9], where we have considered similar problem for the algebra $U_{q_{1}, q_{2}, q_{3}}\left(\widehat{\widehat{\mathfrak{g}} \mathfrak{l}_{1}}\right)$.

The intertwiners we are going to construct are between horizontal Fock representations and vertical vector representations of the quantum toroidal algebra $U_{q_{1}, q_{2}, q_{3}}\left(\widehat{\hat{\mathfrak{g}}}_{N}\right)$. We will see that, just as in the "abelian" $U_{q_{1}, q_{2}, q_{3}}\left(\widehat{\hat{\mathfrak{g l}}}_{1}\right)$ case, these intertwiners are convenient 
building blocks which can be glued together into what we have called a Higgsed network. The idea of the construction is as follows. In [10] the intertwining operators between Fock representations of $U_{q_{1}, q_{2}, q_{3}}\left(\widehat{\mathfrak{g}}_{1}\right)$ have been shown to reproduce refined topological vertex computations, which in turn can be used to compute Nekrasov partition functions [11] of $5 d$ gauge theories on $\mathbb{C}^{2} \times S^{1}$. In these $5 d$ gauge theories one can tune the parameters so that the theory becomes dual to a $3 d$ theory living on the worldvolume of vortices in the Higgs phase. This tuning procedure is known as Higgsing. The Higgsed intertwiners provide an algebraic framework, in which correlators automatically reproduce Higgsed theory partition functions. Since a lot of degrees of freedom decouple during the Higgsing, the representations featuring in the Higgsed intertwiners are "smaller" than the Fock representations from [10]. In these smaller vector representations the states are labelled by integers (cf. with the Fock representations where they are labelled by Young diagrams, or tuples of integers). Since the representations are smaller, the intertwiners are easier to handle and the computations become more transparent. Besides, vector representations allow one to better use the full power of the large automorphism group of the quantum toroidal algebra (for details see [9]).

In the present short note we consider the following variation of the Higgsing procedure. Let the original $5 d$ gauge theory live not on $\mathbb{C}^{2} \times S^{1}$, but on an ALE space $\mathbb{C}^{2} / \mathbb{Z}_{N} \times S^{1}$. In the algebraic language the partition function of the $5 d$ theory corresponds to a network of Fock space intertwiners for the algebra $U_{q_{1}, q_{2}, q_{3}}\left(\widehat{\mathfrak{g l}}_{N}\right)[12] .{ }^{1}$ The tuning of the parameters corresponding to Higgsing can still be performed, and one arrives at a $3 d$ theory on the vortex worldvolume. However, now the theory is "orbifolded": it lives on $\mathbb{C} / \mathbb{Z}_{N} \times S^{1}$ and there is an additional $\mathbb{Z}_{N} R$-symmetry twist when going around the orbifold point. We will obtain the partition function of the orbifolded theory from the network of Higgsed $U_{q_{1}, q_{2}, q_{3}}\left(\widehat{\widehat{\mathfrak{g l}}}_{N}\right)$ intertwiners. It turns out that the form of the partition function and its algebraic properties are quite similar to those of the original theory. The main difference is that the partition function is not just a function, but a vector-valued function, i.e. there are several partition functions labelled by the indices associated with fundamental hypermultiplets (external lines in the intertwiner picture). These indices correspond to discrete $\mathbb{Z}_{N}$-valued charges associated with flavour symmetries of the theory.

The plan of the paper is as follows. We construct the intertwiners in section 2, compute their correlators in section 3 and show how to interpret them in the gauge theory and algebraic language in section 4 . Since, the main ideas have already been laid out in [9], we allow ourselves to be brief and present only the main features of the $\mathfrak{g l}_{N}$ generalization.

In order not to clutter the main part of the paper with technical details, we move the notations and definitions to the appendices. There we define the algebra $U_{q_{1}, q_{2}, q_{3}}\left(\widehat{\mathfrak{\mathfrak { g }}}_{N}\right)$ (section A), list its properties (section A.1, A.2 and A.3), and write down its relevant representations (section A.4, A.5). Section A.6 contains some facts about the correspondence between the $U_{q_{1}, q_{2}, q_{3}}\left(\widehat{\hat{\mathfrak{g l}}}_{N}\right)$ algebra and geometry of a class of toric CY threefolds.

\footnotetext{
${ }^{1}$ More general algebraic models corresponding to the $5 d$ theory on $\mathbb{C}^{2} /\left(\mathbb{Z}_{N_{1}} \times \mathbb{Z}_{N_{2}}\right) \times S^{1}$ have recently been considered [13].
} 


\section{The intertwiners}

Similarly to [9] we would like to build an intertwining operator $\Phi: \mathcal{V}_{q_{1}} \otimes \mathcal{F}_{q_{1}, q_{3}}^{(1,0), k}(u) \rightarrow$ $\mathcal{F}_{q_{1}, q_{3}}^{(1,0), k}\left(q_{3}^{-1} u\right)$. By definition the intertwiner satisfies

$$
g \Phi=\Phi \Delta(g),
$$

where $g$ is any element of $U_{q_{1}, q_{2}, q_{3}}\left(\widehat{\widehat{\mathfrak{g l}}}_{N}\right)$ and $\Delta$ is the coproduct given in appendix A.1. Representations $\mathcal{V}_{q_{1}}$ and $\mathcal{F}_{q_{1}, q_{3}}^{(1,0), k}(u)$ featuring in the intertwiner are defined in appendices A.4 and A.5 respectively. Since there will be only one Fock representation in our story, we omit the indices $q_{1}, q_{3}$ henceforth.

We represent the intertwiner graphically as a trivalent junction:

$$
\Phi_{q_{1}}^{j}(w)=\mathcal{F}^{(1,0), k}\left(q_{3}^{-1} u\right) \underbrace{\mathcal{V}_{q_{1}}|j, w\rangle} \quad \mathcal{F}^{(1,0), k}(u)^{\prime} \quad j=1, \ldots, N .
$$

We denote the vector representation $V_{q_{1}}$ as blue dashed line, while the Fock space is represented by a solid black line. The essential new ingredients compared to the $U_{q_{1}, q_{2}, q_{3}}\left(\widehat{\mathfrak{g l}}_{1}\right)$ case are the $\mathbb{C}^{N}$ index $j$ of the intertwiner and the color $k$ of the horizontal Fock representation. The explicit expression for the intertwiner is

$$
\begin{aligned}
\Phi_{q_{1}}^{j}(w)= & e^{Q_{j}}\left(-q^{-1} q_{1}^{-j} w\right)^{P_{j}} \exp \left[\sum_{n \geq 1} \frac{\left(q_{1}^{-j} w\right)^{n}}{n} q^{-n} a_{j,-n}\right] \\
& \times \exp \left[-\sum_{n \geq 1} \frac{\left(q_{1}^{-j} w\right)^{-n}}{n}\left(q^{n} a_{j, n}+\frac{q^{-n}-q^{n}}{1-q_{1}^{n N}} \sum_{l=0}^{N-1} q_{1}^{n(\overline{l-j})} a_{l, n}\right)\right],
\end{aligned}
$$

where $\overline{l-j}=(l-j) \bmod N$. The definitions of the bosons $a_{j, n}$ and the zero modes $Q_{j}$, $P_{j}$ are given in appendix A.5. Notice that in the "unrefined limit" $q \rightarrow 1$ the second line simplifies, so that $\Phi_{q_{1}}^{j}(w)$ becomes the conventional undeformed free boson vertex operator. The check that eq. (2.2) indeed intertwines the action of the algebra is provided in appendix B.

The dual intertwiner $\Phi_{j}^{*, q_{1}}: \mathcal{F}_{q_{1}, q_{3}}^{(1,0), k}(u) \rightarrow \mathcal{F}_{q_{1}, q_{3}}^{(1,0), k}\left(q_{3} u\right) \otimes \mathcal{V}_{q_{1}}$ is given by ${ }^{2}$

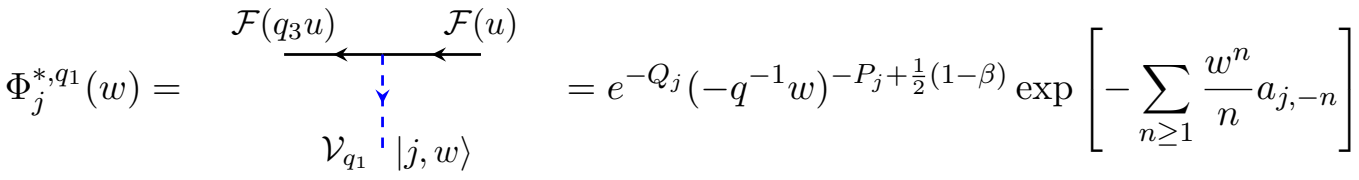

$$
\begin{aligned}
& \times \exp \left[\sum_{n \geq 1} \frac{w^{-n}}{n} q^{n}\left(q^{n} a_{j, n}+\frac{q^{-n}-q^{n}}{1-q_{1}^{n N}} \sum_{l=0}^{N-1} q_{1}^{n(\overline{l-j})} a_{l, n}\right)\right],
\end{aligned}
$$

where $\beta=\frac{\ln q_{3}}{\ln q_{1}}$.

\footnotetext{
${ }^{2}$ In what follows (except in appendix B) we will implicitly shift the argument $w$ of $\Phi_{q_{1}}^{j}(w)$ and $\Phi_{j}^{*, q_{1}}(w)$ by $q_{1}^{j}$ to simplify the expressions.
} 
Due to the reflection symmetry $\rho$ of the algebra (see appendix A.2) there is another pair of intertwiners with the vector representation $\mathcal{V}_{q_{3}}$ (denoted by the dashed red lines):

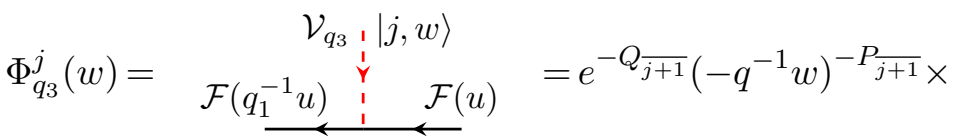

$$
\begin{aligned}
& \times \exp \left[-\sum_{n \geq 1} \frac{w^{n}}{n} d^{-n} q_{2}^{-n}\left(a_{\overline{j+1},-n}+q_{3}^{n} \frac{1-q_{2}^{n}}{1-q_{3}^{n N}} \sum_{l=0}^{N-1}{q_{3}^{\overline{(l-j-2)}}} a_{l,-n}\right)\right] \exp \left[\sum_{n \geq 1} \frac{w^{-n}}{n} d^{n} a_{\overline{j+1, n},}\right],
\end{aligned}
$$

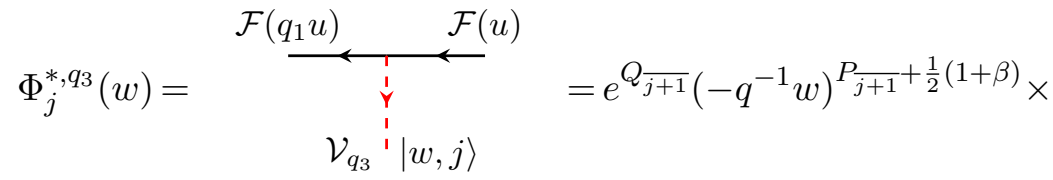

$$
\begin{aligned}
& \times \exp \left[\sum_{n \geq 1} \frac{w^{n}}{n} q_{3}^{n}\left(a_{\overline{j+1},-n}+q_{3}^{n} \frac{1-q_{2}^{n}}{1-q_{3}^{n N}} \sum_{l=0}^{N-1} q_{3}^{\overline{(l-j-2)} n} a_{l,-n}\right)\right] \exp \left[-\sum_{n \geq 1} \frac{w^{-n}}{n} q_{3}^{-n} a_{\overline{j+1}, n}\right] .
\end{aligned}
$$

We will omit some zero modes henceforth since their contributions to the correlators are just powers of the spectral parameters.

\section{Correlators}

Having the explicit form of the intertwining operators (2.3)-(2.6) we can straightforwardly compute their correlators. Moreover, since the intertwiners are built free boson vertex operators Wick theorem applies, so it is sufficient to compute only the pairwise correlators. They are given by

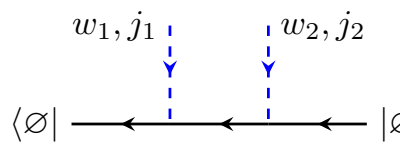

$$
\begin{aligned}
& =\left\langle\varnothing\left|\Phi_{q_{1}, k}^{j_{1}}\left(w_{1}\right) \Phi_{q_{1}, k}^{j_{2}}\left(w_{2}\right)\right| \varnothing\right\rangle \\
& \sim\left(w_{1}-w_{2}\right)^{\delta_{j_{1}, j_{2}}} \frac{\left(q_{1}^{\overline{j_{2}-j_{1}}} q_{2}^{-1} \frac{w_{2}}{w_{1}} ; q_{1}^{N}\right)_{\infty}}{\left(q_{1}^{j_{2}-j_{1}} \frac{w_{2}}{w_{1}} ; q_{1}^{N}\right)_{\infty}},
\end{aligned}
$$

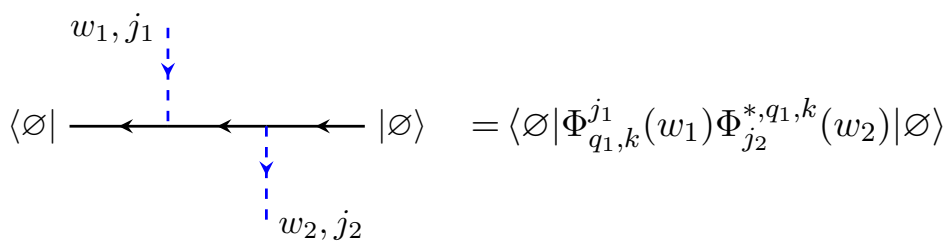

$$
\sim\left(w_{1}-q w_{2}\right)^{-\delta_{j_{1}, j_{2}}} \frac{\left(q_{1}^{\overline{j_{2}-j_{1}}} q \frac{w_{2}}{w_{1}} ; q_{1}^{N}\right)_{\infty}}{\left(q_{1}^{\overline{j_{2}-j_{1}}} q^{-1} \frac{w_{2}}{w_{1}} ; q_{1}^{N}\right)_{\infty}}
$$

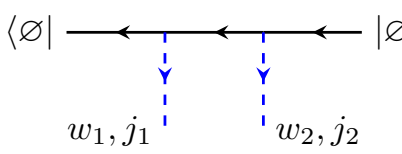

$$
\begin{aligned}
& =\left\langle\varnothing\left|\Phi_{j_{1}}^{*, q_{1}, k}\left(w_{1}\right) \Phi_{j_{2}}^{*, q_{1}, k}\left(w_{2}\right)\right| \varnothing\right\rangle \\
& \sim\left(w_{1}-q_{2} w_{2}\right)^{\delta_{j_{1}, j_{2}}} \frac{\left(q_{1}^{\overline{j_{2}-j_{1}}} \frac{w_{2}}{w_{1}} ; q_{1}^{N}\right)_{\infty}}{\left(q_{1}^{\overline{j_{2}-j_{1}}} q_{2} \frac{w_{2}}{w_{1}} ; q_{1}^{N}\right)_{\infty}},
\end{aligned}
$$




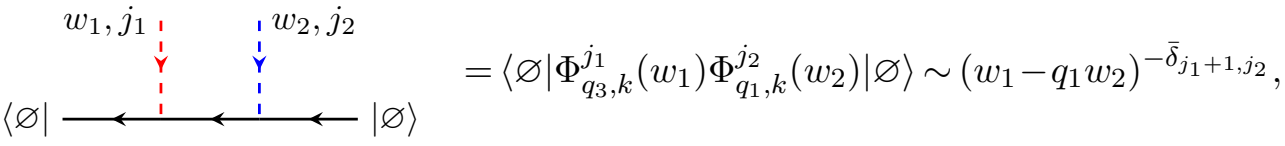

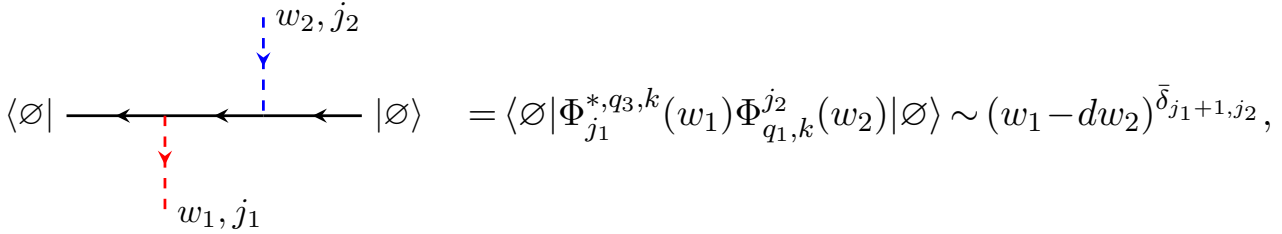

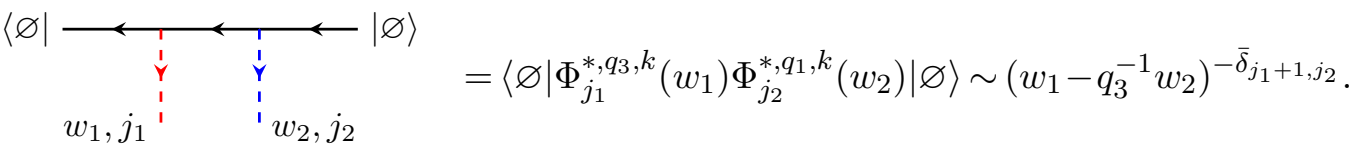

The proportionality signs in eqs. (3.1)-(3.6) are to remind us that we have omitted some simple factors originating from the zero modes. We don't list the correlators of $\mathcal{V}_{q_{3}}$ intertwiner with another $\mathcal{V}_{q_{3}}$ intertwiner, because they are obtained from eqs. (3.1)-(3.3) by the exchange $q_{1} \leftrightarrow q_{3}$ and $j \leftrightarrow \overline{(-j)}$.

\subsection{R-matrix}

Having computed the correlators, we can deduce the commutation relations for the intertwiners. They feature the diagonal $R$-matrix of $U_{q_{1}, q_{2}, q_{3}}\left(\widehat{\hat{\mathfrak{g l}}}_{N}\right)$ acting in the tensor product of two vector representations:

$$
\Phi_{q_{1}, k}^{j_{1}}\left(w_{1}\right) \Phi_{q_{1}, k}^{j_{2}}\left(w_{2}\right)=A\left(\frac{w_{1}}{w_{2}}\right) R_{q_{1} q_{1}}\left(\frac{w_{1}}{w_{2}}\right) \Phi_{q_{1}, k}^{j_{2}}\left(w_{2}\right) \Phi_{q_{1}, k}^{j_{1}}\left(w_{1}\right)
$$

where $A(x)$ is a certain $q_{1}^{N}$-periodic combination of theta-functions and the $R$-matrix is given by

$$
R(x)=\left(\frac{w_{2}-q_{2} w_{1}}{w_{2}-w_{1}}\right)^{\delta_{j_{1}, j_{2}}} \frac{\left(\overline{q_{1}-j_{2}} \frac{w_{1}}{w_{2}} ; q_{1}^{N}\right)_{\infty}^{2}}{\left(q_{1}^{j_{1}-j_{2}} q_{2} \frac{w_{1}}{w_{2}} ; q_{1}^{N}\right)_{\infty}\left(q_{1}^{j_{1}-j_{2}} q_{2}^{-1} \frac{w_{1}}{w_{2}} ; q_{1}^{N}\right)_{\infty}}
$$

Similarly, we learn that

$$
\Phi_{j_{1}}^{*, q_{1}, k}\left(w_{1}\right) \Phi_{j_{2}}^{*, q_{1}, k}\left(w_{2}\right)=B\left(\frac{w_{1}}{w_{2}}\right) \frac{1}{R_{q_{1} q_{1}}\left(\frac{w_{1}}{w_{2}}\right)} \Phi_{j_{2}}^{*, q_{1}, k}\left(w_{2}\right) \Phi_{j_{1}}^{*, q_{1}, k}\left(w_{1}\right)
$$

where $B(x)$ is another $q_{1}^{N}$-periodic combination. The dual intertwiners commute (up to a $q_{1}^{N}$-periodic function $\left.C(x)\right)$

$$
\Phi_{j_{1}}^{*, q_{1}, k}\left(w_{1}\right) \Phi_{q_{1}, k}^{j_{2}}\left(w_{2}\right)=C\left(\frac{w_{1}}{w_{2}}\right) \Phi_{q_{1}, k}^{j_{2}}\left(w_{2}\right) \Phi_{j_{1}}^{*, q_{1}, k}\left(w_{1}\right)
$$

We don't pay attention to the $q_{1}^{N}$-periodic functions since in the partition function we are going to study in the next section the intertwiners are going to be under the $q_{1}^{N}$-Jackson integral (or, equivalently, under contour integral with $q_{1}^{N}$-periodic strings of poles). 


\section{Screenings and networks}

Similarly to [9] we can build a network of intertwiners by combining them either vertically or horizontally. We have considered all possible horizontal combinations in the previous section. In this section we combine the intertwiners vertically to tie together several horizontal solid lines.

\subsection{Screening operators}

Two possible vertical combinations of intertwiners give rise to two screening currents acting in the tensor product of two Fock spaces:

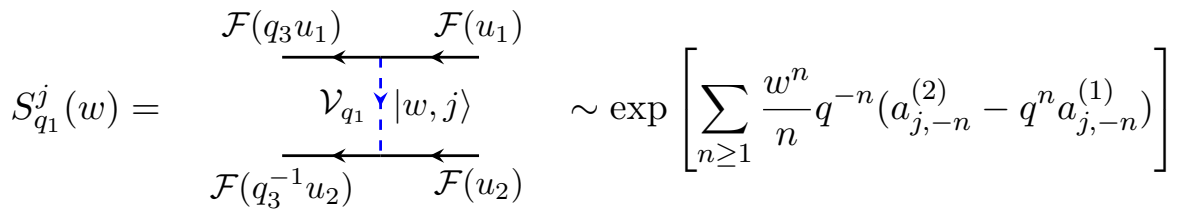

$$
\begin{aligned}
& \times \exp \left[-\sum_{n \geq 1} \frac{w^{-n}}{n}\left(q^{n}\left(a_{j, n}^{(2)}-q^{n} a_{j, n}^{(1)}\right)+\frac{q^{-n}-q^{n}}{1-q_{1}^{n N}} \sum_{l=0}^{N-1} q_{1}^{n(\overline{l-j})}\left(a_{l, n}^{(2)}-q^{n} a_{l, n}^{(1)}\right)\right)\right],
\end{aligned}
$$

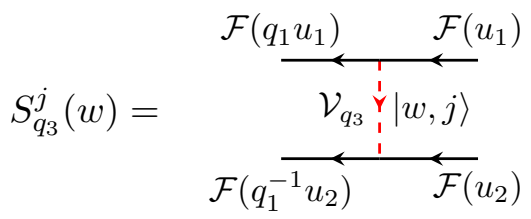

$$
\begin{aligned}
& \sim \exp \left[-\sum_{n \geq 1} \frac{w^{n}}{n} d^{-n} q_{2}^{-n}\left(\left(a_{j+1,-n}^{(2)}-q^{n} a_{j+1,-n}^{(1)}\right)\right.\right. \\
& \left.\left.+q_{3}^{n} \frac{1-q_{2}^{n}}{1-q_{3}^{n N}} \sum_{l=0}^{N-1} q_{3}^{\overline{(l-j-2)} n}\left(a_{l,-n}^{(2)}-q^{n} a_{\overline{j+1},-n}^{(1)}\right)\right)\right] \\
& \times \exp \left[\sum_{n \geq 1} \frac{w^{-n}}{n} d^{n}\left(a_{\frac{(2)}{j+1, n}}-q^{n} a_{\frac{(1)}{j+1, n}}\right)\right],
\end{aligned}
$$

where $a_{i, n}^{(1)}$ and $a_{i, n}^{(2)}$ act on the first and the second Fock spaces respectively. If we sum over a complete basis of states in the intermediate vector representations in eqs. (4.1), (4.2) we will get intertwining operators acting in the tensor product of two Fock spaces:

$$
\begin{aligned}
Q_{q_{1}} & =\sum_{j=0}^{N-1} \int \frac{d_{q_{1}^{N}} w}{w} S_{q_{1}}^{j}(w)=\sum_{j=0}^{N-1} \oint \frac{d w}{w} S_{q_{1}}^{j}(w), \\
Q_{q_{3}} & =\sum_{j=0}^{N-1} \oint \frac{d_{q_{1}^{N}} w}{w} S_{q_{3}}^{j}(w)=\sum_{j=0}^{N-1} \oint \frac{d w}{w} S_{q_{3}}^{j}(w)
\end{aligned}
$$

In other words, integrated combinations like $Q_{q_{1}}$ and $Q_{q_{3}}$ are screening charges commuting with the action of the whole $U_{q_{1}, q_{2}, q_{3}}\left(\widehat{\hat{\mathfrak{g l}}}_{N}\right)$ algebra. We can use these screening operators to build " $\mathfrak{g l}_{N}$-extended" $W_{k}$ algebras acting on the tensor product of $k N$ Fock spaces. ${ }^{3}$ By Fock spaces here we mean representations of a single boson, and denote them by $\mathfrak{F}$

\footnotetext{
${ }^{3}$ A version of spectral duality can be used to exchange $k$ and $N$ as was demonstrated in [19].
} 
to distinguish from the Fock representation $\mathcal{F}$ of $U_{q_{1}, q_{2}, q_{3}}\left(\widehat{\hat{\mathfrak{g}}}_{N}\right)$ which is generated by $N$ bosons. To build the $W_{k}$-algebra one needs to cluster together $N$-tuples of Fock spaces:

$$
\mathfrak{F}^{\otimes k N} \simeq\left(\mathfrak{F}^{\otimes N}\right)^{\otimes k} \simeq \mathcal{F}^{\otimes k} .
$$

There are $k$ tuples, on which the screening charges $Q_{q_{1}}^{(a)}$ and $Q_{q_{3}}^{(a)}(a=1, \ldots, k-1)$ can act. The screenings $Q_{q_{1}}^{(a)}$ and $Q_{q_{3}}^{(a)}$ act between $a$-th and $(a+1)$-th $N$-tuple and the $W_{k}$-algebra is generated by the operators commuting with all the screenings. There is a constructive way to obtain the generators: one can simply take a coproduct of any element of $U_{q_{1}, q_{2}, q_{3}}\left(\widehat{\hat{\mathfrak{g l}}}_{N}\right)$ and evaluate it on the tensor product of $k$ Fock spaces. It will automatically commute with the screening charges. As an example of the " $\mathfrak{g l}_{N}$-extended" $q$-deformed $W_{2}$ generators we take $\Delta\left(E_{i}(z)\right)$ (see appendices A.1 and A.5 for the definition of the coproduct and the Fock representation respectively):

$$
\begin{aligned}
& \left.\Delta\left(E_{i}(z)\right)\right|_{\mathcal{F}^{k_{1}}\left(u_{1}\right) \otimes \mathcal{F}^{k_{2}}\left(u_{2}\right)}=e^{\delta_{i, k_{1}} P^{(1)}} e^{Q_{i}^{(1)}-Q_{i+1}^{(1)}} z^{P_{i}^{(1)}-P_{i+1}^{(1)}+1}(-d)^{P^{(1)}} \\
& \times \exp \left[\sum_{n \geq 1} \frac{z^{n}}{n} q^{-n}\left(a_{i,-n}^{(1)}-q_{1}^{-n} a_{\frac{1}{i+1},-n}\right)\right] \\
& \times \exp \left[-\sum_{n \geq 1} \frac{z^{-n}}{n} q^{-n}\left(a_{i, n}^{(1)}-q_{3}^{-n} a_{i+1, n}^{(1)}\right)\right] \\
& +e^{\delta_{i, k_{2}} P^{(2)}} e^{Q_{i}^{(2)}-Q_{\frac{(2)}{i+1}}^{(2)}}(q z)^{P_{i}^{(2)}-P_{\overline{i+1}}^{(2)}+1}(-d)^{P_{\overline{i+1}}^{(2)}} q^{-P_{i}^{(1)}+P_{\bar{i}+1}^{(1)}} \times \\
& \times \exp \left[\sum_{n \geq 1} \frac{z^{n}}{n}\left(a_{i,-n}^{(2)}-q_{1}^{-n} a_{i+1,-n}^{(2)}-\left(q^{n}-q^{-n}\right) q^{\frac{n}{2}}\left(a_{i,-n}^{(1)}-q_{1}^{-n} a_{i+1,-n}^{(1)}\right)\right)\right] \\
& \times \exp \left[-\sum_{n \geq 1} \frac{z^{-n}}{n} q_{2}^{-n}\left(a_{i, n}^{(2)}-q_{3}^{-n} a_{i+1, n}^{(2)}\right)\right] .
\end{aligned}
$$

The operators (4.6) should reproduce the web $W$-algebras [25-28] associated with brane diagram of the form shown in figure 1.

\subsection{Networks and partition functions}

The network partition functions are built from the screening charges in the same way as in the $U_{q_{1}, q_{2}, q_{3}}\left(\widehat{\mathfrak{g l}}_{1}\right)$ case: one draws the solid "warp threads" and then connects them with arbitrary number of blue and red "weft threads". Let us give a simple example of the construction:

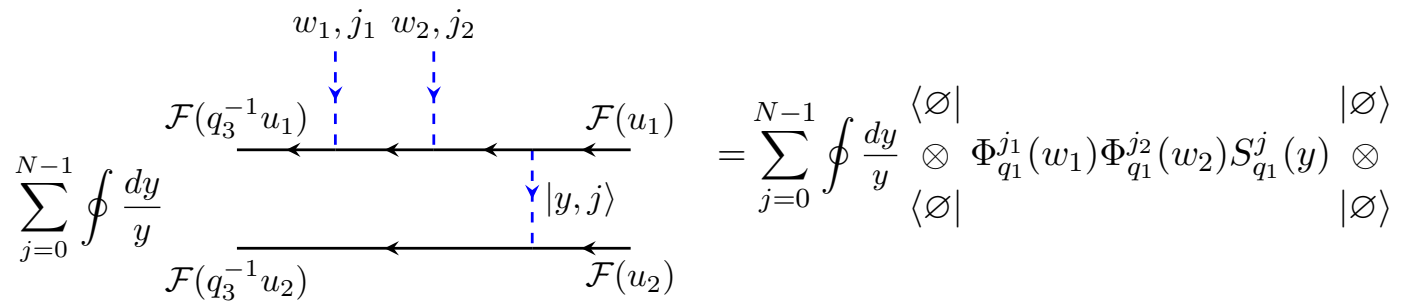

$$
\begin{aligned}
& \sim\left(w_{1}-w_{2}\right)^{\delta_{j_{1}, j_{2}}} \frac{\left(q_{1}^{\overline{j_{2}-j_{1}}} q_{2}^{-1} \frac{w_{2}}{w_{1}} ; q_{1}^{N}\right)_{\infty}}{\left(q_{1}^{\overline{j_{2}-j_{1}}} \frac{w_{2}}{w_{1}} ; q_{1}^{N}\right)_{\infty}} \sum_{j=0}^{N-1} \oint \frac{d y}{y} y^{\log _{q_{2}}} \frac{u_{2}}{u_{1}} \prod_{a=1}^{2}\left(w_{a}-q y\right)^{-\delta_{j_{a}, j}} \frac{\left(q_{1}^{\overline{j-j}} q \frac{y}{w_{a}} ; q_{1}^{N}\right)_{\infty}}{\left(q_{1}^{\overline{j-j a}} q^{-1} \frac{y}{w_{a}} ; q_{1}^{N}\right)_{\infty}} .
\end{aligned}
$$


The integral (4.7) reproduces the partition function of the orbifolded $3 d \mathcal{N}=2^{*} \mathrm{U}(1)$ theory with two hypermultiplets. The localization calculation for this type of theories has been performed in $[29,30]^{4}$ (there $2 d$ versions of the partition functions such as (4.7) has been computed, but the $3 d$ uplift is completely analogous).

The logic of the identification with gauge theories is similar to [9]. One views the picture (4.7) as a brane diagram of Type IIB string theory, so that the horizontal lines are identified with NS5 branes and the vertical dashed lines are D3 branes. The finite D3 brane segment gives rise to a U(1) gauge theory, while the semi-infinite D3 branes represent the hypermultiplets. The remaining directions of the D3 and NS5 branes are then subject to the $\mathbb{Z}_{N}$ orbifolding. The FI, mass and equivariant parameters of the $3 d$ theory are identified with the spectral parameters of the branes: $\log _{q_{1}} \frac{u_{1}}{u_{2}}$ is the FI parameter, $w_{1,2}$ are exponentiated masses of the fundamental hypermultiplets, $q_{2}$ is the real adjoint mass, and $q_{1}$ is the equivariant parameter of the $3 d \Omega$-background (because of the $\mathbb{Z}_{N}$ projection the Pochhammer symbols in (4.7) contain $N$-th power of $q_{1}$ ). The essentially new feature of the $\mathbb{Z}_{N}$ orbifolding is that one needs to choose the discrete $\mathbb{Z}_{N}$ charges of each Coulomb branch parameter and mass of fundamental field in the theory. In the partition function (4.7) the charges are $j$ for the Coulomb branch parameter $y$ and $j_{1,2}$ for the two fundamental masses $w_{1,2}$.

A more general example is that of $\mathrm{U}(n)$ theory with $m$ fundamental hypermultiplets. To get the partition function we just add more dashed lines to the diagram in (4.7):

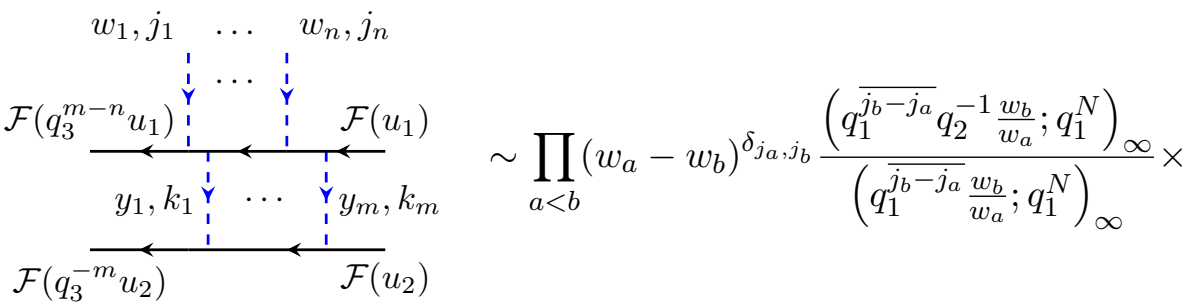

$$
\begin{aligned}
& \times \sum_{\left\{k_{a}\right\}=0}^{N-1} \oint d^{n} y \prod_{k=1}^{N} y_{k}^{\log _{q_{2}} \frac{u_{2}}{u_{1}}} \Delta_{\mathbb{Z}_{N}}^{q_{1}, q_{2}}(\vec{y}, \vec{k}) \prod_{a=1}^{m} \prod_{b=1}^{n}\left(w_{a}-q y_{b}\right)^{-\delta_{j_{a}, k_{b}}} \frac{\left(q_{1}^{\overline{k_{b}-j_{a}}} q \frac{y_{b}}{w_{a}} ; q_{1}^{N}\right)_{\infty}}{\left(q_{1}^{\overline{k_{b}-j_{a}}} q^{-1} \frac{y_{k}}{w_{a}} ; q_{1}^{N}\right)_{\infty}}
\end{aligned}
$$

where the "orbifolded" $\left(q_{1}, q_{2}\right)$-deformed Vandermonde determinant is given by

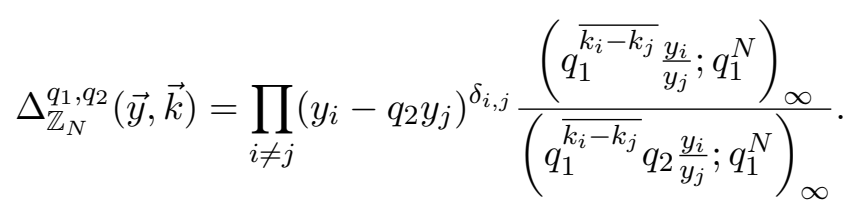

Of course, one can add the blue and red dashed lines together to the picture and obtain partition functions of two orbifolded $3 d$ theories living respectively on

$$
\mathbb{C}_{q_{1}} / \mathbb{Z}_{N} \times S^{1} \subset \mathbb{C}_{q_{1}, q_{3}}^{2} / \mathbb{Z}_{N} \times S^{1}
$$

and

$$
\mathbb{C}_{q_{3}} / \mathbb{Z}_{N} \times S^{1} \subset \mathbb{C}_{q_{1}, q_{3}}^{2} / \mathbb{Z}_{N} \times S^{1}
$$

\footnotetext{
${ }^{4}$ See also earlier work [31, 32] on localization of vortex partition functions without orbifolding.
} 
and coupled through a $1 d$ theory on $S^{1}$. We limit ourselves to a simple example of two coupled $\mathrm{U}(1)$ theories each with a single hypermultiplet:

$$
\begin{aligned}
& \underbrace{}_{y_{1}, k_{1} \dot{y}} \underbrace{w_{1}, j_{1} w_{2}, j_{2}}_{y_{2}, k_{2}} \sim\left(w_{1}-q_{1} w_{2}\right)^{-\bar{\delta}_{j_{1}+1, j_{2}}} \sum_{j=0}^{N-1} \oint d^{2} y\left(y_{1} y_{2}\right)^{\log _{q_{2}} \frac{u_{2}}{u_{1}}}\left(y_{1}-q_{1} y_{2}\right)^{-\bar{\delta}_{j_{1}+1, j_{2}}} \\
& \times\left(y_{1}-q_{3}^{-1} y_{2}\right)^{-\bar{\delta}_{j_{1}+1, j_{2}}}\left(y_{1}-d w_{2}\right)^{-\bar{\delta}_{k_{1}+1, j_{2}}}\left(y_{2}-d w_{1}\right)^{-\bar{\delta}_{k_{2}+1, j_{1}}}
\end{aligned}
$$

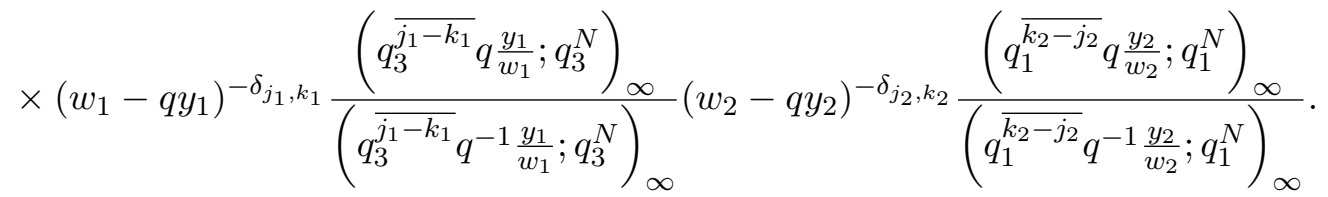

\section{Conclusions}

In this short note we have presented the natural generalization of the Higgsed network formalism to "non-abelian" quantum toroidal algebras $U_{q_{1}, q_{2}, q_{3}}\left(\widehat{\hat{\mathfrak{g}}}_{N}\right)$. We have found the intertwiners ("Higgsed vertices"), computed their correlators, and built screening operators from them. The correlators of intertwiners are related to partition functions of $3 d$ gauge theories on orbifolded spacetimes.

There are several further questions about the models we have considered. The partition functions we have obtained should satisfy a version of $(q, t)-\mathrm{KZ}$ equation [12], and they should also be related to the eignefunctions of a spin generalization of the RuijsenaarsSchneider integrable system. It would be interesting to clarify the interplay between these two systems.

An even more interesting, but also apparently more difficult task, is to obtain general bosonization formulas for toroidal algebras. The bosonization currently known for $U_{q_{1}, q_{2}, q_{3}}\left(\widehat{\hat{\mathfrak{g l}}}_{N}\right)$ is the Fock representation [14, 15], which is written in terms of $N$ free bosons and therefore has fixed central charge $C_{1}=q$. It is thus the toroidal analogue of the Frenkel-Kac construction [20, 21] for affine Lie algebras. This bosonization also follows naturally from the geometric action of $U_{q_{1}, q_{2}, q_{3}}\left(\widehat{\widehat{\mathfrak{g l}}}_{N}\right)$ on the moduli space of instantons [22]. A general bosonization formula for $U_{q_{1}, q_{2}, q_{3}}\left(\widehat{\hat{\mathfrak{g l}}}_{N}\right)$, if it exists, should involve $N^{2}$ free bosons and produce arbitrary central charges. This would be an analogue of the Wakimoto bosonization [23] for affine Lie algebras.

As we briefly explain in appendix A.6, quantum toroidal algebras $U_{q_{1}, q_{2}, q_{3}}\left(\widehat{\hat{\mathfrak{g l}}}_{N}\right)$ are related to toric Calabi-Yau three-folds of the form shown in figure 1. More generally, one can consider an arbitrary toric strip geometry, i.e. a manifold whose toric diagram has $N$ semi-infinite horizontal lines attached from the right and $M$ lines attached from the left with no loops. This geometry corresponds to a quantum toroidal superalgebra $U_{q_{1}, q_{2}, q_{3}}\left(\widehat{\hat{\mathfrak{g l}}}_{N \mid M}\right)$ recently introduced in [24]. It should be straightforward to generalize our results to this case. An interesting conjecture is that any toric CY threefold corresponds to a quantum algebra, which is a "two-dimensional" generalization of quantum toroidal 
algebras. The gluing approach recently popularized in [25-28] could probably help to define these general algebras.

\section{Acknowledgments}

The author is partly supported by the RSF grant 18-71-10073.

\section{A The algebra $U_{q_{1}, q_{2}, q_{3}}\left(\widehat{\widehat{\mathfrak{g l}}}_{N}\right)$}

In this appendix we collect the definitions related to the algebra $U_{q_{1}, q_{2}, q_{3}}\left(\widehat{\hat{\mathfrak{g}}}_{N}\right)$. We mainly follow [16]. To simplify the presentation we limit ourselves to the cases when $N \geq 3$, since the $N=2$ case requires special treatment. We use the standard notations for the quantum parameters of the algebra ${ }^{5}$

$$
q_{1}=\frac{d}{q}, \quad q_{2}=q^{2}, \quad q_{3}=\frac{1}{q d}
$$

The algebra $U_{q_{1}, q_{2}, q_{3}}\left(\widehat{\mathfrak{g l}}_{N}\right)$ is generated by modes of the currents:

$$
\begin{aligned}
E_{i}(z) & =\sum_{n \in \mathbb{Z}} E_{i, n} z^{-n}, \quad F_{i}(z)=\sum_{n \in \mathbb{Z}} F_{i, n} z^{-n} \\
K_{i}^{ \pm}(z) & =K_{i}^{ \pm 1} \pm \sum_{n \geq 1} K_{i, \pm n} z^{\mp n}=K_{i}^{ \pm 1} \exp \left[ \pm\left(q-q^{-1}\right) \sum_{n \geq 1} H_{i, \pm n} z^{-n}\right]
\end{aligned}
$$

with $i=0, \ldots, N-1$ and the central element $C_{1}$ satisfying the following commutation relations: 6

$$
\begin{aligned}
& K_{i}^{ \pm}(z) K_{j}^{ \pm}(w)=K_{j}^{ \pm}(w) K_{i}^{ \pm}(z), \\
& \frac{g_{i, j}\left(C_{1}^{-1} z, w\right)}{g_{i, j}\left(C_{1} z, w\right)} K_{i}^{-}(z) K_{j}^{+}(w)=\frac{g_{i, j}\left(w, C_{1}^{-1} z\right)}{g_{i, j}\left(w, C_{1} z\right)} K_{j}^{+}(w) K_{i}^{-}(z), \\
& d_{i, j} g_{i, j}(z, w) K_{i}^{+}(z) E_{j}(w)+g_{j, i}(w, z) E_{j}(w) K_{i}^{+}(z)=0, \\
& d_{i, j} g_{i, j}(z, w) K_{i}^{-}\left(C_{1} z\right) E_{j}(w)+g_{j, i}(w, z) E_{j}(w) K_{i}^{-}\left(C_{1} z\right)=0, \\
& d_{j, i} g_{j, i}(w, z) K_{i}^{+}\left(C_{1} z\right) F_{j}(w)+g_{i, j}(z, w) F_{j}(w) K_{i}^{+}\left(C_{1} z\right)=0, \\
& d_{j, i} g_{j, i}(w, z) K_{i}^{-}(z) F_{j}(w)+g_{i, j}(z, w) F_{j}(w) K_{i}^{-}(z)=0, \\
& {\left[E_{i}(z), F_{j}(z)\right]=\frac{\delta_{i, j}}{q-q^{-1}}\left(\delta\left(C_{1} \frac{w}{z}\right) K_{i}^{+}(z)-\delta\left(C_{1} \frac{z}{w}\right) K_{i}^{-}(w)\right),} \\
& d_{i, j} g_{i, j}(z, w) E_{i}(z) E_{j}(w)+g_{j, i}(w, z) E_{j}(w) E_{i}(z)=0, \\
& d_{j, i} g_{j, i}(w, z) F_{i}(z) F_{j}(w)+g_{i, j}(z, w) F_{j}(w) F_{i}(z)=0,
\end{aligned}
$$

\footnotetext{
${ }^{5}$ In [9] we have used a less symmetric notation $U_{q, t}\left(\widehat{\hat{\mathfrak{g l}}}_{1}\right)$ for $U_{q_{1}, q_{2}, q_{3}}\left(\widehat{\widehat{\mathfrak{g l}}}_{1}\right)$, so that $q_{[9]}=\left(q_{1}\right)$ here and $t_{[9]}=\left(q_{2}\right)_{\text {here }}^{-1}$.

${ }^{6}$ We conform with the conventions of $[12,16]$. The relations given in $[17,18]$ are obtained from the convention we use by the redefinition $\left(K_{i}^{ \pm}(z)\right)_{[17,18]}=\left(K_{i}^{ \pm}\left(C_{1}^{1 / 2} z\right)\right)_{\text {here }}$.
} 


$$
\begin{aligned}
& {\left[E_{i}\left(z_{1}\right),\left[E_{i}\left(z_{2}\right), E_{i \pm 1}(w)\right]_{q}\right]_{q^{-1}}+\left[E_{i}\left(z_{2}\right),\left[E_{i}\left(z_{1}\right), E_{i \pm 1}(w)\right]_{q}\right]_{q^{-1}}=0} \\
& {\left[F_{i}\left(z_{1}\right),\left[F_{i}\left(z_{2}\right), F_{i \pm 1}(w)\right]_{q}\right]_{q^{-1}}+\left[F_{i}\left(z_{2}\right),\left[F_{i}\left(z_{1}\right), F_{i \pm 1}(w)\right]_{q}\right]_{q^{-1}}=0}
\end{aligned}
$$

where

$$
\begin{aligned}
{[A, B]_{q} \stackrel{\text { def }}{=} A B-q B A, } \\
g_{i, j}(z, w)=\left(z-d^{-m_{i, j}} q^{a_{i, j}} w\right)= \begin{cases}z-q_{2} w, & i=j \bmod N, \\
z-q_{1} w, & i=j-1 \bmod N, \\
z-q_{3} w, & i=j+1 \bmod N, \\
z-w, & \text { otherwise },\end{cases} \\
d_{i, j}=d^{m_{i, j}}= \begin{cases}d^{\mp 1}, & i=j \mp 1 \bmod N, \\
1, & \text { otherwise }\end{cases}
\end{aligned}
$$

The second central element of the algebra is given by the product of the zero modes $K_{i}$ :

$$
C_{2}=\prod_{i=0}^{N-1} K_{i} .
$$

Sometimes it will be more convenient for us to write the relations directly for the modes of the generating currents:

$$
\begin{aligned}
& {\left[K_{i}, K_{j}\right]=0, \quad\left[K_{i}, H_{j, n}\right]=0,} \\
& K_{i} E_{j, n}=q^{a_{i, j}} E_{j, n} K_{i}, \quad K_{i} F_{j, n}=q^{-a_{i, j}} F_{j, n} K_{i}, \\
& {\left[H_{i, n}, H_{j, m}\right]=\frac{d^{-n m_{i, j}}\left(q^{n a_{i, j}}-q^{-n a_{i, j}}\right)\left(C_{1}^{n}-C_{1}^{-n}\right)}{n\left(q-q^{-1}\right)^{2}} \delta_{n+m, 0},} \\
& {\left[H_{i, n}, E_{j, m}\right]=d^{-n m_{i, j}} C_{1}^{\frac{n-|n|}{2}} \frac{\left(q^{n a_{i, j}}-q^{-n a_{i, j}}\right)}{n\left(q-q^{-1}\right)} E_{j, n+m},} \\
& {\left[H_{i, n}, F_{j, m}\right]=-d^{-n m_{i, j}} C_{1}^{\frac{n+|n|}{2}} \frac{\left(q^{n a_{i, j}}-q^{-n a_{i, j}}\right)}{n\left(q-q^{-1}\right)} F_{j, n+m},} \\
& {\left[E_{i, n}, F_{j, m}\right]=\delta_{i, j}\left(1-\delta_{n+m, 0}\right) \frac{K_{i, n+m} C_{1}^{-m \theta_{n+m}>0-n \theta_{n+m<0}}}{q-q^{-1}}+\delta_{i, j} \delta_{n+m, 0} \frac{C_{1}^{n} K_{i}-C_{1}^{-n} K_{i}^{-1}}{q-q^{-1}}} \\
& d^{m_{i, j}}\left(E_{i, n+1} E_{j, m}-q^{a_{i, j}} E_{j, m} E_{i, n+1}\right)-\left(q^{a_{i, j}} E_{i, n} E_{j, m+1}-E_{j, m+1} E_{i, n}\right)=0, \\
& d^{m_{i, j}}\left(F_{i, n+1} F_{j, m}-q^{-a_{i, j}} F_{j, m} F_{i, n+1}\right)-\left(q^{-a_{i, j}} F_{i, n} F_{j, m+1}-F_{j, m+1} F_{i, n}\right)=0, \\
& {\left[E_{i, n},\left[E_{i, m}, E_{i \pm 1, k}\right]_{q}\right]_{q^{-1}}+\left[E_{i, m},\left[E_{i, n}, E_{i \pm 1, k}\right]_{q}\right]_{q^{-1}}=0,} \\
& {\left[F_{i, n},\left[F_{i, m}, F_{i \pm 1, k}\right]_{q}\right]_{q^{-1}}+\left[F_{i, m},\left[F_{i, n}, F_{i \pm 1, k}\right]_{q}\right]_{q^{-1}}=0,}
\end{aligned}
$$

where $\theta_{n>0}$ gives 1 if $n>0$ or 0 if $n \leq 0$.

The algebra is doubly graded with the first grading $d_{1}$ counting the number of the mode of a current:

$$
\left[d_{1}, E_{i, n}\right]=n E_{i, n}, \quad\left[d_{1}, F_{i, n}\right]=n F_{i, n}, \quad\left[d_{1}, H_{i, n}\right]=n H_{i, n}, \quad\left[d_{1}, K_{i}\right]=\left[d_{1}, C_{1}\right]=0 .
$$


The second grading $d_{2}$ is given by

$$
\left[d_{2}, E_{i, n}\right]=\delta_{i, 0} E_{i, n}, \quad\left[d_{2}, F_{i, n}\right]=-\delta_{i, 0} F_{i, n}, \quad\left[d_{2}, H_{i, n}\right]=0, \quad\left[d_{2}, K_{i}\right]=\left[d_{2}, C_{1}\right]=0 .
$$

There are two natural quantum affine subalgebras $U_{q}\left(\widehat{\mathfrak{g l}}_{N}\right)$ inside $U_{q_{1}, q_{2}, q_{3}}\left(\widehat{\hat{\mathfrak{g l}}}_{N}\right)$, the "horizontal" and the "vertical" ones. The horizontal subalgebra $U_{q}\left(\widehat{\mathfrak{g l}}_{N}\right)_{\text {hor }}$ is generated by the zero modes of the currents $E_{i, 0}, F_{i, 0}, K_{i}$. Notice that $i=0, \ldots, N-1$ labels the roots of the affine root system $\widehat{A}_{N}$. This gives the "traceless" subalgebra $U_{q}\left(\widehat{\mathfrak{s l}}_{N}\right)_{\text {hor }} \subset U_{q}\left(\widehat{\mathfrak{g l}}_{N}\right)_{\text {hor }}$. An additional horizontal Heisenberg subalgebra is generated by the sequential commutators of non-zero modes, e.g. $E_{0,1}$ and $E_{i,-1}$ or $E_{0,-1}$ and $E_{i, 1}$. The horizontal subalgebra has vanishing $d_{1}$ grading, while $d_{2}$ counts the modes of the quantum affine currents.

The vertical quantum affine subalgebra $U_{q}\left(\widehat{\mathfrak{g l}}_{N}\right)_{\text {vert }}$ is obtained by forgetting about the currents $E_{0}(z), F_{0}(z)$. The remaining currents $E_{i}(z), F_{i}(z), K_{i}^{ \pm}(z)$ with $i=1, \ldots, N-1$ are Drinfeld currents for the "traceless part" $U_{q}\left(\widehat{\mathfrak{s l}}_{N}\right)_{\text {vert }} \subset U_{q}\left(\widehat{\mathfrak{g l}}_{N}\right)_{\text {vert }}$ of the vertical subalgebra. Adding $K_{0}^{ \pm}(z)$ gives an extra Heisenberg subalgebra. The role of the gradings $d_{1}$ and $d_{2}$ is reversed in the vertical subalgebra compared to the horizontal one.

\section{A.1 Coalgebra structure}

The algebra $U_{q_{1}, q_{2}, q_{3}}\left(\widehat{\widehat{\mathfrak{g l}}}_{N}\right)$ can be given a coalgebra structure with the coproduct structure given by

$$
\begin{aligned}
\Delta\left(E_{i}(z)\right) & =E_{i}(z) \otimes 1+K_{i}^{-}\left(C_{1}^{(1)} z\right) \otimes E_{i}\left(C_{1}^{(1)} z\right), \\
\Delta\left(F_{i}(z)\right) & =F_{i}\left(C_{1}^{(2)} z\right) \otimes K_{i}^{+}\left(C_{1}^{(2)} z\right)+1 \otimes F_{i}(z), \\
\Delta\left(K_{i}^{+}(z)\right) & =K_{i}^{+}(z) \otimes K_{i}^{+}\left(\left(C_{1}^{(1)}\right)^{-1} z\right), \\
\Delta\left(K_{i}^{-}(z)\right) & =K_{i}^{-}\left(\left(C_{1}^{(2)}\right)^{-1} z\right) \otimes K_{i}^{-}(z), \\
\Delta\left(C_{1}\right) & =C_{1} \otimes C_{1},
\end{aligned}
$$

where $C_{1}^{(1)}=C_{1} \otimes 1, C_{1}^{(2)}=1 \otimes C_{1}$. As in the $U_{q_{1}, q_{2}, q_{3}}\left(\widehat{\hat{\mathfrak{g l}}}_{1}\right)$ case, there is an infinite number of equivalent but not identical coalgebra structures, parametrized by splittings of the algebra into pairs of Borel subalgebras. The coproducts corresponding to different coalgebra structures are related by Drinfeld twists. For definiteness we call the coproduct (A.31)-(A.35) vertical, since it acts (almost) diagonally on the vertical currents $K_{i}^{ \pm}(z)$ (A.33), (A.34).

\section{A.2 Symmetries of the algebra}

The algebra $U_{q_{1}, q_{2}, q_{3}}\left(\widehat{\hat{\mathfrak{g l}}}_{N}\right)$ has a large group of symmetries including:

1. $\mathrm{SL}(2, \mathbb{Z})$ automorphisms. The generator $S$ of this group is also known as the Miki's automorphism, which, in particular, transforms the horizontal quantum affine subalgebra into the vertical one. The central charges $C_{1}$ and $C_{2}$ and the gradings $d_{1}$ and $d_{2}$ are also exchanged by the automorphism $S$. The $\operatorname{SL}(2, \mathbb{Z})$ duality group is shared by all known quantum toroidal algebras including the simplest one, $U_{q_{1}, q_{2}, q_{3}}\left(\widehat{\mathfrak{g l}}_{1}\right)$, because it originates from the mapping class group of the torus. 
2. Reflection symmetry. This transformation, which we call $\rho$, exchanges the deformation parameters $q_{1}$ and $q_{3}$, and reflects the $\widehat{A}_{N}$ indices of the generating currents:

$$
\begin{gathered}
\rho: U_{q_{1}, q_{2}, q_{3}}\left(\widehat{\hat{\mathfrak{g l}}}_{N}\right) \rightarrow U_{q_{3}, q_{2}, q_{1}}\left(\widehat{\widehat{\mathfrak{g l}}}_{N}\right), \\
\rho\left(E_{i}(z)\right)=E_{(-i) \bmod N}(z), \quad \rho\left(F_{i}(z)\right)=F_{(-i) \bmod N}(z), \quad \rho\left(H_{i}(z)\right)=H_{(-i) \bmod N}(z) .
\end{gathered}
$$

Evidently $\rho^{2}=1$, so the reflection symmetry generates $\mathfrak{S}_{2}$. This symmetry is smaller, than the corresponding symmetry of the $U_{q_{1}, q_{2}, q_{3}}\left(\widehat{\hat{\mathfrak{g l}}}_{1}\right)$, in which case any the permutation of $\left(q_{1}, q_{2}, q_{3}\right)$ was allowed (besides, no relabelling of the generators was needed).

3. The symmetries listed so far were already present in the $U_{q_{1}, q_{2}, q_{3}}\left(\widehat{\widehat{\mathfrak{g}}}_{1}\right)$ algebra. In the $\mathfrak{g l}_{N}$ case there is one more important automorphism $\sigma$, rotating the roots of $\widehat{A}_{N-1}$ root system:

$$
\sigma\left(E_{i}(z)\right)=E_{i+1 \bmod N}(z), \quad \sigma\left(F_{i}(z)\right)=F_{i+1 \bmod N}(z) \quad \sigma\left(K_{i}(z)\right)=K_{i+1 \bmod N}(z) .
$$

Evidently, $\sigma^{N}=1$. The central charges $C_{1}$ and $C_{2}$ are invariant under the symmetry, and so is the $d_{1}$ grading. The $d_{2}$ grading is modified by the symmetry, but in a controlled way. Let $\delta_{i}$ be the grading associated with root $i$ :

$$
\begin{aligned}
{\left[\delta_{i}, E_{j, n}\right] } & =\delta_{i, j} E_{j, n}, & {\left[\delta_{i}, F_{j, n}\right] } & =-\delta_{i, j} F_{j, n}, \\
{\left[\delta_{i}, H_{j, n}\right] } & =0, & {\left[\delta_{i}, K_{j}\right] } & =\left[\delta_{i}, C_{1}\right]=0,
\end{aligned}
$$

so that $\delta_{0}=d_{2}$. Then

$$
\sigma\left(\delta_{i}\right)=\delta_{i+1 \bmod N}
$$

\section{A.3 The limit of $U_{q_{1}, q_{2}, q_{3}}\left(\widehat{\widehat{g l}}_{N}\right)$ to $W_{1+\infty}\left[\mathfrak{g l}_{N}\right]$ and the quantum torus}

In the limit $q_{2} \rightarrow 1$ the algebra $U_{q_{1}, q_{2}, q_{3}}\left(\widehat{\widehat{\mathfrak{g l}}}_{N}\right)$ can be described very explicitly as the central extension of the algebra of matrix-valued functions on the quantum torus [16]. The quantum torus $T_{d^{N}}^{2}$ is a non-commutative space with coordinates ${ }^{7} x$ and $p$ satisfying

$$
p x=d^{-N} x p
$$

with $d=q_{1}=q_{3}^{-1}$ playing the role of the parameter of the noncommutativity. The algebra of matrix-valued functions is generated by

$$
W_{i, j}^{(n, m)}=d^{-N \frac{n m}{2}} e_{i, j} x^{n} p^{m}, \quad m, n \in \mathbb{Z}, \quad i, j=1, \ldots, N,
$$

where $e_{i, j}$ is a basis in the space of $N \times N$ matrices:

$$
\left(e_{i, j}\right)_{k, l}=\delta_{i, k} \delta_{j, l} \quad i, j, k, l=1, \ldots, N .
$$

\footnotetext{
${ }^{7}$ We always assume that the coordinates are complexified.
} 
After the central extension with two central charges $c_{1}$ and $c_{2}$, the generators $W_{i, j}^{(n, m)}$ satisfy the following Lie algebra relations:

$$
\begin{aligned}
{\left[W_{i, j}^{(n, m)}, W_{k, l}^{(r, s)}\right]=} & \delta_{j, k} d^{-N \frac{m r-n s}{2}} W_{i, l}^{(n+r, m+s)}-\delta_{i, l} d^{N \frac{m r-n s}{2}} W_{k, j}^{(n+r, m+s)} \\
& +\delta_{n+r, 0} \delta_{m+s, 0} \delta_{j, k} \delta_{i, l}\left(n c_{1}+m c_{2}\right) .
\end{aligned}
$$

Notice that the relations (A.43) are explicitly covariant under the $\operatorname{SL}(2, \mathbb{Z})$ transformations acting on the upper indices of the generators $W_{i, j}^{(n, m)}$, while the central charges $c_{1}$ and $c_{2}$ transform as a doublet. The algebra (A.43) is also called $W_{1+\infty}\left[\mathfrak{g l}_{N}\right]$.

The generating currents of $U_{q_{1}, q_{2}, q_{3}}\left(\widehat{\hat{\mathfrak{g l}}}_{N}\right)$ are given by the formulas

$$
\begin{aligned}
E_{i, n} & = \begin{cases}W_{i, i+1}^{(n, 0)} d^{-i n}, & i=1, \ldots, N-1, \\
W_{N, 1}^{(n, 1)} d^{-n \frac{N}{2}}, & i=0,\end{cases} \\
F_{i, n} & = \begin{cases}W_{i+1, i}^{(n, 0)} d^{-i n}, & i=1, \ldots, N-1, \\
W_{1, N}^{(n,-1)} d^{-n \frac{N}{2}}, & i=0,\end{cases} \\
H_{i, n} & = \begin{cases}\left(W_{i, i}^{(n, 0)}-W_{i+1, i+1}^{(n, 0)} d^{-i n},\right. & i=1, \ldots, N-1, \\
\left(d^{-n N} W_{N, N}^{(n, 0)}-W_{1,1}^{(n, 0)}\right)+c_{2} \delta_{n, 0}, & i=0,\end{cases} \\
c_{1,2}= & \lim _{q_{2} \rightarrow 1} \log _{q_{2}} C_{1,2} .
\end{aligned}
$$

\section{A.4 Vector representations of $U_{q_{1}, q_{2}, q_{3}}\left(\widehat{\widehat{(g l}}_{N}\right)$}

Vector representation $\mathcal{V}_{q_{1}}$ is a representation of $U_{q_{1}, q_{2}, q_{3}}\left(\widehat{\hat{\mathfrak{g l}}}_{N}\right)$ on the space of $\mathbb{C}^{N}$-valued functions of a single variable $x$. It has trivial central charges, $C_{1}=C_{2}=1$. We can take as basis states of this representation tensor products of Dirac delta functions and basis vectors $\mathbf{e}_{j}$ in $\mathbb{C}^{N}$. We write them as ${ }^{8}$

$$
|u, j\rangle=\delta\left(q_{1}^{j} \frac{x}{u}\right) \mathbf{e}_{j}, \quad j=1, \ldots, N
$$

In eq. (A.48) we view the spectral parameter $u$ as the parameter of the state, not of the representations (the latter convention was adopted in $[16,18])$. These two views are in

\footnotetext{
${ }^{8}$ Our notation for the states of the vector representation is related to that of [16] by the formula

$$
\left([u]_{j}^{(k)}\right)_{[16]}=\left(\left|q_{1}^{j-k} u,(k-j-1) \bmod N+1\right\rangle\right)_{\text {here }}, \quad j \in \mathbb{Z},
$$
}

so that

$$
\begin{aligned}
E_{i}(z)[u]_{j}^{(k)} & =\delta\left(q_{1}^{j+1} \frac{u}{z}\right) \bar{\delta}_{i+j+1, k}[u]_{j+1}^{(k)}, \\
F_{i}(z)[u]_{j}^{(k)} & =\delta\left(q_{1}^{j} \frac{u}{z}\right) \bar{\delta}_{i+j, k}[u]_{j-1}^{(k)}, \\
K_{i}^{ \pm}(z)[u]_{j}^{(k)} & =\psi^{\bar{\delta}_{i+j, k}}\left(q_{1}^{j} \frac{u}{z}\right) \psi^{-\bar{\delta}_{i+j+1, k}}\left(q_{1}^{j} q_{3}^{-1} \frac{u}{z}\right)[u]_{j}^{(k)} .
\end{aligned}
$$


fact completely equivalent. The algebra $U_{q_{1}, q_{2}, q_{3}}\left(\widehat{\mathfrak{g}}_{N}\right)$ acts on the states (A.48) as matrices valued in difference operators ${ }^{9}$ in $x$ :

$$
\begin{aligned}
E_{i}(z) & = \begin{cases}e_{N, 1} \delta\left(\frac{x}{z}\right) q_{1}^{-x \partial_{x}}, & i=0, \\
e_{i, i+1} \delta\left(\frac{x}{z}\right) q_{1}^{-x \partial_{x}}, & i=1, \ldots, N-1,\end{cases} \\
F_{i}(z) & = \begin{cases}e_{1, N} \delta\left(q_{1} \frac{x}{z}\right) q_{1}^{x \partial_{x}}, & i=0, \\
e_{i+1, i} \delta\left(q_{1} \frac{x}{z}\right) q_{1}^{x \partial_{x}}, & i=1, \ldots, N-1,\end{cases} \\
K_{i}^{ \pm}(z) & = \begin{cases}\psi^{e_{N, N}}\left(\frac{x}{z}\right) \psi^{-e_{1,1}}\left(q_{3}^{-1} \frac{x}{z}\right), & i=0, \\
\psi^{e_{i, i}}\left(\frac{x}{z}\right) \psi^{-e_{i+1, i+1}}\left(q_{3}^{-1} \frac{x}{z}\right), & i=1, \ldots, N-1,\end{cases}
\end{aligned}
$$

where

$$
\psi(x)=\sqrt{q_{2}} \frac{1-q_{2}^{-1} x}{1-x}=q_{2}^{-\frac{1}{2}} \frac{1-q_{2} x^{-1}}{1-x^{-1}},
$$

and $e_{i, j}$ are basis matrices defined in eq. (A.42). The eigenvalues of the currents $K^{+}(z)$ and $K^{-}(z)$ are given by the same rational function understood as a series expansion around $z=0$ and $z=\infty$ respectively. Let us also write the zero modes $K_{i}$ explicitly:

$$
K_{i}= \begin{cases}q_{2}^{\frac{e_{N, N}-e_{1,1}}{2}}, & i=0 \\ q_{2}^{\frac{e_{i, i}-e_{i+1, i+1}}{2}}, & i=1, \ldots, N-1\end{cases}
$$

so that indeed $C_{2}=\prod_{i=0}^{N-1} K_{i}=1$.

Due to the symmetry $\rho$ of the algebra (eq. (A.36)) there exists a representation $\mathcal{V}_{q_{3}}$ defined similarly to eq. (A.49)-(A.51) with $q_{1} \leftrightarrow q_{3}$ and the labels of the generators reversed.

In [16] a set of $N$ different vector representations $\mathcal{V}_{q_{1}}^{(k)}$ related to each other by the action of $\sigma$ has been defined. Due to $\sigma$ being the symmetry of the algebra, all the representations in the family are isomorphic. Here we make do with just one representation $\mathcal{V}_{q_{1}}$. We do so by noticing that $\sigma$ can be represented on the space of vector-valued functions as a cyclic permutation matrix $s$ :

$$
\sigma(g)|u, j\rangle=s g s^{-1}|u, j\rangle
$$

where

$$
s=\sum_{i=1}^{N-1} e_{i+1, i}+e_{1, N} .
$$

In this way all $N$ representations $\mathcal{V}_{q_{1}}^{(k)}$ are obtained from $\mathcal{V}_{q_{1}}=\mathcal{V}_{q_{1}}^{(0)}$ by the shift of the indices.

\footnotetext{
${ }^{9}$ Note that the action of the shift operator in $x$ on a state $|u, j\rangle$ can be understood as an inverse shift of the parameter $u$ :

$$
q_{1}^{x \partial_{x}}|u, j\rangle=\left|q_{1}^{-1} u, j\right\rangle .
$$
}




\section{A.5 Horizontal Fock representation of $U_{q_{1}, q_{2}, q_{3}}\left(\widehat{\widehat{\mathfrak{g l}}}_{N}\right)$}

In the horizontal Fock representation $[14,15] \mathcal{F}_{q_{1}, q_{3}}^{(1,0), k}(u)$ all currents of the $U_{q_{1}, q_{2}, q_{3}}\left(\widehat{\mathfrak{g}}_{N}\right)$ algebra are expressed through vertex operators built from the generators $H_{i, n}$ constituting $N$ copies of the Heisenberg algebra (see eq. (A.21)). The central charges of $\mathcal{F}_{q_{1}, q_{3}}^{(1,0), k}(u)$ are $C_{1}=q=\sqrt{q_{2}}$ and $C_{2}=1$. The representation $\mathcal{F}_{q_{1}, q_{3}}^{(1,0), k}(u)$ is characterized by a spectral parameter $u \in \mathbb{C}^{\times}$and the color index $k=0, \ldots, N-1$.

We define the fundamental vertex operators

$$
V_{i}^{ \pm}(z)=\exp \left[\mp \sum_{n \geq 1} \frac{q-q^{-1}}{q^{n}-q^{-n}} H_{i, \pm n} z^{\mp n}\right]
$$

Notice that $V^{ \pm}(z)$ contain only positive or negative modes respectively and are therefore automatically normal ordered. The product of $V^{-}(z) V^{+}(w)$ is normal ordered too, but $V^{+}(z) V^{-}(w)$ is not, and for it we have the following identity:

$$
V_{i}^{(+)}(z) V_{j}^{(-)}(w)=s_{i j}(z, w) V_{j}^{(-)}(w) V_{i}^{(+)}(z), \quad|z|>|w|,
$$

where

$$
s_{i j}(z, w)=\frac{\left(1-\frac{q w}{z}\right)^{\bar{\delta}_{i, j}}\left(1-\frac{w}{q z}\right)^{\bar{\delta}_{i, j}}}{\left(1-\frac{d w}{z}\right)^{\bar{\delta}_{i, j-1}}\left(1-\frac{w}{d z}\right)^{\bar{\delta}_{i, j+1}}}
$$

where $\bar{\delta}_{i, j}$ gives one if $i=j \bmod N$ and zero otherwise. Using $V^{ \pm}(z)$ we define the vertex operator representation of $U_{q_{1}, q_{2}, q_{3}}\left(\widehat{\hat{\mathfrak{g l}}}_{N}\right)$ :

$$
\begin{aligned}
E_{i}(z) & =\eta_{i}(z) e^{\bar{\alpha}_{i}} z^{H_{i, 0}+1} e^{\bar{\delta}_{i, k} P}, \\
F_{i}(z) & =\xi_{i}(z) e^{-\bar{\alpha}_{i}} z^{-H_{i, 0}+1} e^{-\bar{\delta}_{i, k} P}, \\
K_{i}^{ \pm}\left(q^{\frac{1}{2}} z\right) & =\varphi_{i}^{ \pm}(z) q^{ \pm H_{i, 0}},
\end{aligned}
$$

where $P$ is the momentum operator giving $\ln u$ on the whole space $\mathcal{F}_{q_{1}, q_{3}}^{(1,0), k}(u)$ (this definition seems superfluous when we are dealing with a single Fock representation, but will be convenient when we will study tensor products of Fock representations). The vertex operators $\eta_{i}(z), \xi_{i}(z)$ and $\phi_{i}^{ \pm}(z)$ are given by:

$$
\begin{aligned}
\eta_{i}(z) & =V_{i}^{-}\left(q^{-\frac{1}{2}} z\right) V_{i}^{+}\left(q^{\frac{1}{2}} z\right), \\
\xi_{i}(z) & =\left(V_{i}^{-}\left(q^{\frac{1}{2}} z\right)\right)^{-1}\left(V_{i}^{+}\left(q^{-\frac{1}{2}} z\right)\right)^{-1}, \\
\varphi_{i}^{ \pm}(z) & =V_{i}^{ \pm}\left(q^{ \pm 1} z\right)\left(V_{i}^{ \pm}\left(q^{\mp 1} z\right)\right)^{-1},
\end{aligned}
$$


and $\bar{\alpha}_{i}, H_{i, 0}(i=0, \ldots, N-1)$ are the zero modes satisfying the relations ${ }^{10}$

$$
\begin{aligned}
e^{\bar{\alpha}_{i}} e^{\bar{\alpha}_{j}} & =(-d)^{-m_{i, j}} e^{\bar{\alpha}_{j}} e^{\bar{\alpha}_{i}}, \\
z^{H_{i, 0}} e^{\bar{\alpha}_{j}} & =z^{a_{i, j}} e^{\bar{\alpha}_{j}} z^{H_{i, 0}}, \\
{\left[H_{i, 0}, H_{j, 0}\right] } & =0 .
\end{aligned}
$$

The relation (A.68) can be understood as a $\mathbb{C}^{\times}$central extension of the group algebra of the $\widehat{A}_{N-1}$ root lattice. ${ }^{11}$ Notice that $\sum_{i=0}^{N-1} \bar{\alpha}_{i}$ and $\sum_{i=0}^{N-1} H_{i, 0}$ are central elements of the zero mode algebra (A.68)-(A.70). Since for $\mathcal{F}_{q_{1}, q_{3}}^{(1,0), k}(u)$ the central charge $C_{2}=1$, we have to fix

$$
\sum_{i=0}^{N-1} H_{i, 0}=0 .
$$

We also set

$$
\sum_{i=0}^{N-1} \bar{\alpha}_{i}=0
$$

Due to eqs. (A.71), (A.72) we can understand $H_{i, 0}$ as measuring discrete "momenta" taking values in the weight lattice of $A_{N-1}$ and $e^{\bar{\alpha}_{i}}$ as the generators shifting these momenta by simple roots of $A_{N-1}$.

Thus, the states in the Fock representation are generated by $N$ free bosons $H_{i, n}$ for $n \neq 0$, and in addition by the zero modes $e^{\bar{\alpha}_{i}}$. A general state is labelled by an $N$-tuple of Young diagrams $\vec{Y}$ and a weight $\bar{\mu}$ of the algebra $\mathfrak{s l}_{N}$. We denote this state by $\left|u, e^{\bar{\mu}}, \vec{Y}\right\rangle$. The vacuum state of the representation $\mathcal{F}_{q_{1}, q_{3}}^{(1,0), k}(u)$ is

$$
\left|u, e^{\bar{\omega}_{k}}, \vec{\varnothing}\right\rangle
$$

where $\bar{\omega}_{k}$ is the $k$-th fundamental weight of $A_{N-1}$.

Independent Heisenberg generators. We find it convenient to introduce another basis $a_{i, n}$ in $N$ copies of Heisenberg algebra spanned by $H_{i, n}(i=0, \ldots, N-1)$. We write

$$
H_{i, n}=\frac{\left(q^{n}-q^{-n}\right)}{n\left(q-q^{-1}\right)} q^{-\frac{|n|}{2}}\left(a_{i, n}-q^{|n|} d^{n} a_{\overline{i+1, n}}\right)
$$

where $\bar{i}=i \bmod N$, so that bosons $a_{i, n}$ are completely independent:

$$
\left[a_{i, n}, a_{j, m}\right]=n \delta_{n+m, 0} \delta_{i, j} .
$$

\footnotetext{
${ }^{10}$ Our relations for the zero modes differ slightly from the relations found in $[12,14,15]$. There three types of zero modes $\bar{\alpha}_{i}, H_{i, 0}, \partial_{\bar{\alpha}_{i}}$ satisfying

$$
\begin{aligned}
e^{\bar{\alpha}_{i}} e^{\bar{\alpha}_{j}} & =(-1)^{a_{i, j}} e^{\bar{\alpha}_{j}} e^{\bar{\alpha}_{i}}, \\
{[12,14,15]: \quad z^{\partial_{\bar{\alpha}_{i}}} e^{\bar{\alpha}_{j}} } & =z^{a_{i, j}} e^{\bar{\alpha}_{j}} z^{\partial_{\bar{\alpha}_{i}}}, \\
z^{H_{i, 0}} e^{\bar{\alpha}_{j}} & =z^{a_{i, j}} d^{-\frac{1}{2} m_{i, j}} e^{\bar{\alpha}_{j}} z^{H_{i, 0}}
\end{aligned}
$$

have been used. Notice that the power notation $z^{H_{i, 0}}$ in this case is somewhat misleading, since eq. (A.67) is inconsistent with $z_{1}^{H_{i, 0}} z_{2}^{H_{i, 0}}=\left(z_{1} z_{2}\right)^{H_{i, 0}}$.

${ }^{11}$ This is an obvious generalization the $\mathbb{Z}_{2}$ central extension, which is used in conventional vertex operator algebras.
} 
We can also simplify the relations (A.68), (A.70) by introducing zero modes $Q_{i}, P_{i}$, satisfying the canonical commutation relations

$$
\left[P_{i}, Q_{j}\right]=\delta_{i, j}, \quad\left[Q_{i}, Q_{j}\right]=\left[P_{i}, P_{j}\right]=0, \quad i, j=0, \ldots, N-1 .
$$

The zero modes $e^{Q_{i}}, H_{i, 0}$ are expressed through $Q_{i}, P_{i}$ as follows:

$$
e^{\bar{\alpha}_{i}}=e^{Q_{i}-Q_{\overline{i+1}}}(-d)^{P_{\overline{i+1}}}, \quad H_{i, 0}=P_{i}-P_{\overline{i+1}} .
$$

In terms of $a_{i, n}, Q_{i}, P_{i}$ we have the following expression for the vertex operators (A.62)(A.64) (similar formulas appeared in [19]):

$$
\begin{aligned}
E_{i}(z)= & e^{\delta_{i, k} P} e^{Q_{i}-Q_{\overline{i+1}}} z^{P_{i}-P_{\overline{i+1}}+1}(-d)^{P_{\overline{i+1}}} \exp \left[\sum_{n \geq 1} \frac{z^{n}}{n} q^{-n}\left(a_{i,-n}-q_{1}^{-n} a_{\overline{i+1},-n}\right)\right] \\
& \times \exp \left[-\sum_{n \geq 1} \frac{z^{-n}}{n} q^{-n}\left(a_{i, n}-q_{3}^{-n} a_{\overline{i+1}, n}\right)\right] \\
F_{i}(z)= & e^{-\delta_{i, k} P} e^{-Q_{i}+Q_{\overline{i+1}}} z^{-P_{i}+P_{\overline{i+1}}+1}(-d)^{-P_{\overline{i+1}}} \exp \left[-\sum_{n \geq 1} \frac{z^{n}}{n}\left(a_{i,-n}-q_{1}^{-n} a_{\overline{i+1},-n}\right)\right] \\
& \times \exp \left[\sum_{n \geq 1} \frac{z^{-n}}{n}\left(a_{i, n}-q_{3}^{-n} a_{\overline{i+1}, n}\right)\right], \\
K_{i}^{+}(z)= & q^{P_{i}-P_{\overline{i+1}}} \exp \left[\sum_{n \geq 1} \frac{z^{-n}}{n}\left(q^{n}-q^{-n}\right) q^{-\frac{n}{2}}\left(a_{i, n}-q_{3}^{-n} a_{\overline{i+1}, n}\right)\right], \\
K_{i}^{-}(z)= & q^{-P_{i}+P_{\overline{i+1}}} \exp \left[-\sum_{n \geq 1} \frac{z^{n}}{n}\left(q^{n}-q^{-n}\right) q^{-\frac{n}{2}}\left(a_{i,-n}-q_{1}^{-n} a_{\overline{i+1},-n}\right)\right]
\end{aligned}
$$

Apparently, for $U_{q_{1}, q_{2}, q_{3}}\left(\widehat{\hat{\mathfrak{g}}}_{N}\right)$ only one type of Fock representations $\mathcal{F}_{q_{1}, q_{3}}^{(1,0), k}(u)$ with central charge $C_{1}=\sqrt{q_{2}}$ is known. $\mathcal{F}_{q_{1}, q_{3}}^{(1,0), k}(u)$ is invariant under the reflection symmetry $\rho$ (A.36). The rotation symmetry $\sigma$ shifts the color index $k$ of the representations by one modulo $N$. This situation is in contrast to $U_{q_{1}, q_{2}, q_{3}}\left(\widehat{\widehat{\mathfrak{g l}}}_{1}\right)$, where there where three Fock representations $\mathcal{F}_{q_{a}, q_{b}}^{(1,0), k}(u)$ with $a \neq b$ and central charges $C_{1}=\sqrt{q_{c}}$, where $c \neq a \neq b$. Of course, since $q_{2}$ plays a distinguished role in the algebra $U_{q_{1}, q_{2}, q_{3}}\left(\widehat{\hat{\mathfrak{g l}}}_{N}\right)$, the fact that the representation with central charge related to $q_{2}$ is also distinguished in this case is not surprising. Still, we think that this point deserves further investigation.

\section{A.6 Branching into $U_{q_{1}, q_{2}, q_{3}}\left(\widehat{\widehat{\mathfrak{g l}}}_{1}\right)$ algebras and toric geometry}

There is a geometric way of viewing the algebra $U_{q_{1}, q_{2}, q_{3}}\left(\widehat{\widehat{\mathfrak{g l}}}_{N}\right)$, which can be helpful for understanding its representations and subalgebras. The algebra $U_{q_{1}, q_{2}, q_{3}}\left(\widehat{\widehat{\mathfrak{g l}}}_{N}\right)$ is associated with a toric Calabi-Yau three-fold of the form $X_{N} \times \mathbb{C}$, where $X_{N}=\widetilde{\mathbb{C}^{2} / \mathbb{Z}_{N}}$ is the blowup of the $A_{N}$ singularity. There is a $\mathbb{C}^{3}$ action on the toric $\mathrm{CY}$; denote its weights by $q_{1}, q_{2}$, $q_{3}$. We turn off the $\mathbb{C}^{\times}$part of $\mathbb{C}^{3}$ which scales the canonical bundle, so the weights satisfy 

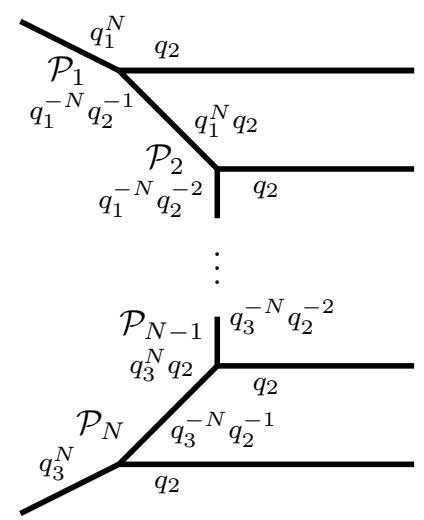

Figure 1. Toric diagram of the threefold $X_{N} \times \mathbb{C}$, where $X_{N}=\widetilde{\mathbb{C}^{2} / \mathbb{Z}_{N}}$. Each trivalent vertex corresponds to a $\left(\mathbb{C}^{\times}\right)^{3}$ fixed point $\mathcal{P}_{a}$ with indices on the legs denoting the weights of the $\left(\mathbb{C}^{\times}\right)^{3}$ action.

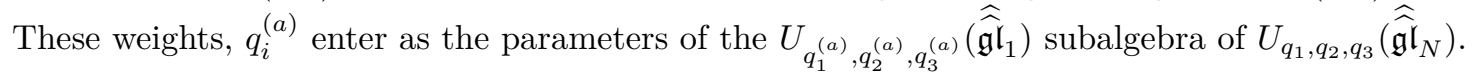

$q_{1} q_{2} q_{3}=1$. We choose the weights so that $q_{2}$ scales the trivial $\mathbb{C}$ fiber, while the choice of $q_{1}$ is easier to shown on the toric diagram (figure 1).

Let us notice that for $N=1$ the threefold is simply $\mathbb{C}^{3}$ with weights $q_{i}$ rotating the coordinate planes $\mathbb{C}$. It is therefore logical to assume that the neighbourhoods of the fixed points $\mathcal{P}_{a}$ of the $\left(\mathbb{C}^{\times}\right)^{3}$ action inside $X_{N} \times \mathbb{C}$ might be related to $U_{q_{1}^{(a)}, q_{2}^{(a)}, q_{3}^{(a)}}\left(\widehat{\hat{\mathfrak{g l}}}_{1}\right)$ subalgebras inside $U_{q_{1}, q_{2}, q_{3}}\left(\widehat{\hat{\mathfrak{g l}}}_{N}\right)$. Having accepted this hypothesis we see that the deformation parameters $q_{i}^{(a)}$ should be related to the weights of the fixed planes joining at the fixed point $a$. They are fixed by the geometry of the threefold (see figure 1):

$$
q_{1}^{(a)}=q_{1}^{N} q_{2}^{a-1}, \quad q_{2}^{(a)}=q_{2}, \quad q_{3}^{(a)}=q_{1}^{-N} q_{2}^{-a}, \quad a=1, \ldots, N .
$$

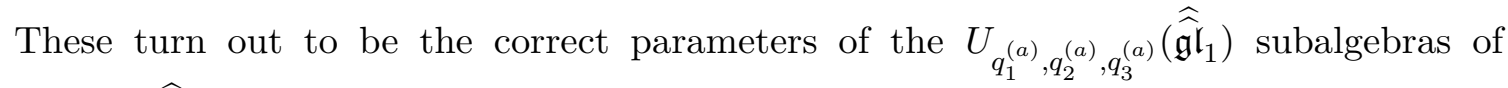
$U_{q_{1}, q_{2}, q_{3}}\left(\widehat{\widehat{\mathfrak{g l}}}_{N}\right)$ described in [16]. These subalgebras are easier to understand in the $q_{2} \rightarrow 1$ limit. In this limit, described in appendix A.3, the algebra $U_{q_{1}, q_{2}, q_{3}}\left(\widehat{\hat{\mathfrak{g}}}_{N}\right)$ turns into the central extension of the algebra of matrix-valued functions on the quantum torus $T_{d^{N}}^{2}$.

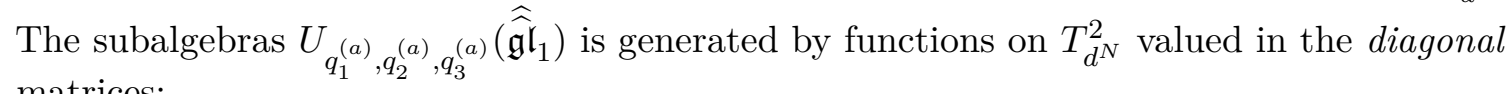
matrices:

$$
U_{q_{1}^{(a)}, q_{2}^{(a)}, q_{3}^{(a)}}\left(\widehat{\mathfrak{g l}}_{1}\right)=\left\langle W_{a, a}^{(n, m)}\right\rangle, \quad n, m \in \mathbb{Z} .
$$

Notice also that in this limit $q_{i}^{(a)}$ are independent of $a$ :

$$
q_{1}^{(a)}=q_{1}^{N}=d^{N}, \quad q_{2}^{(a)}=1, \quad q_{3}^{(a)}=q_{1}^{-N}=d^{-N}, \quad \text { for } q_{2} \rightarrow 1 .
$$

One can also deduce some larger subalgebras of $U_{q_{1}, q_{2}, q_{3}}\left(\widehat{\hat{\mathfrak{g l}}}_{N}\right)$ using the geometric picture. Indeed, let us cut the lowest vertex from the diagram in figure 1 . We are left with the CY threefold $X_{N-1} \times \mathbb{C}$, which inherits the $\left(\mathbb{C}^{\times}\right)^{3}$ action from $X_{N} \times \mathbb{C}$. The corresponding subalgebra of $U_{q_{1}, q_{2}, q_{3}}\left(\widehat{\hat{\mathfrak{g l}}}_{N}\right)$ is $U_{q_{1}^{\prime}, q_{2}^{\prime}, q_{3}^{\prime}}\left(\widehat{\hat{\mathfrak{g l}}}_{N-1}\right)$ with

$$
q_{1}^{\prime}=q_{1}^{1+\frac{1}{N-1}}, \quad q_{2}^{\prime}=q_{2}, \quad q_{3}^{\prime}=q_{3} q_{1}^{-\frac{1}{N-1}} .
$$


This subalgebra in the $q_{2} \rightarrow 1$ limit corresponds to $(N-1) \times(N-1)$ matrix-valued functions on the quantum torus with the $(N-1) \times(N-1)$ block embedded into $N \times N$ matrices in the upper left corner. More generally by cutting the toric diagram from figure 1 one can get subalgebras corresponding to $k \times k$ blocks inside an $N \times N$ matrix sitting on the diagonal. The parameters are easily deduces from the picture.

Thus the structure of the toric skeleton of the CY threefold is related to certain natural subalgebras of $U_{q_{1}, q_{2}, q_{3}}\left(\widehat{\hat{\mathfrak{g l}}}_{N}\right)$. The correspondence we have described originates from the geometric representation theory. In particular, the $U_{q_{1}, q_{2}, q_{3}}\left(\widehat{\hat{\mathfrak{g l}}}_{N}\right)$ algebra acts by correspondences on the equivariant $K$-theory of the moduli space of instantons on $X_{N}$. The correspondences can be seen as adding or removing instantons from the theory. In the equivariant setup the instantons are effectively of zero size and concentrate near fixed points of the equivariant action. Hence, there are subsets of correspondences adding or removing instantons only near some fixed points. These subsets turns out to be a subalgebras.

A more physical argument for cutting the toric diagram might be to notice that the action on the $K$-theory is independent of the area of the two-cycles inside $X_{N}$, so one can scale the area of some cycles to become infinitely large, in which case the fixed points separated by these two-cycles stop talking to each other.

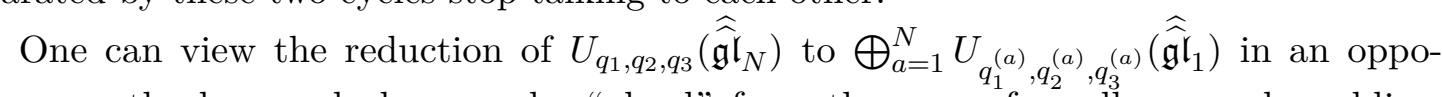
site way: the larger algebra can be "glued" from the sum of smaller ones by adding

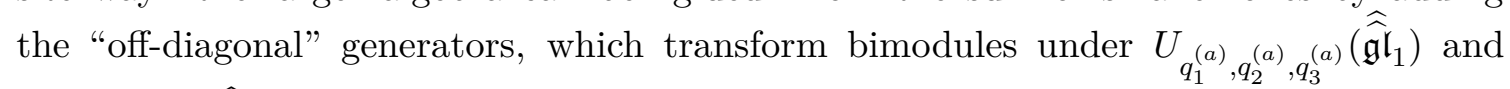

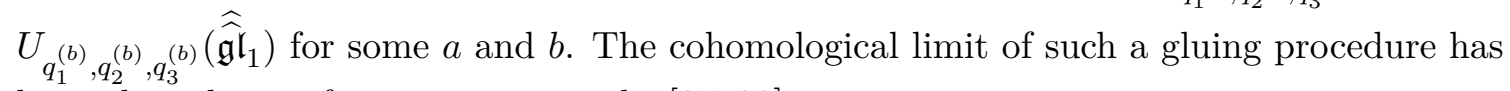
been the subject of many recent works [25-28].

\section{B Intertwining property of the higgsed vertex}

In this appendix we demonstrate intertwining properties of the Higgsed vertices. To do this one needs to check the fundamental property $(2.1)$ of the vertex $\Phi: \mathcal{V}_{q_{1}} \otimes \mathcal{F}_{q_{1}, q_{3}}^{(1,0), k}(u) \rightarrow$ $\mathcal{F}_{q_{1}, q_{3}}^{(1,0), k}\left(q_{3}^{-1} u\right.$ ) (and a similar one for $\Phi^{*}$ ), which has to hold for any element $g \in U_{q_{1}, q_{2}, q_{3}}\left(\widehat{\hat{\mathfrak{g l}}}_{N}\right)$.

The calculations are rather technical, so to keep our account brief we write down explicitly only the check for the current $g=E_{i}(z)$. The coproduct of $E_{i}(z)$ is given by eq. (A.31). In the case at hand the first factor in the tensor product acts on the vector representation $\mathcal{V}_{q_{1}}$, while the second acts on the Fock representation $\mathcal{F}_{q_{1}, q_{3}}^{(1,0), k}(u)$. Thus, the central charge $C_{1}^{(1)}=C_{1} \otimes 1$ is trivial. Using the explicit forms of the currents $E_{i}(z)$ (eq. (A.49)) and $K_{i}^{-}(z)$ (eq. (A.51)) in the vector representation, we write the intertwining property (2.1) which we need to check as

$$
\begin{aligned}
\left.E_{i}(z)\right|_{\mathcal{F}_{q_{1}, q_{3}}^{(1,0), k}(u)} \Phi_{q_{1}}^{j}(w) & \stackrel{?}{=} \delta_{i, \bar{j}-1} \delta\left(\frac{w}{q_{1}^{j-1} z}\right) \Phi_{q_{1}}^{\overline{j-1}}(w)+ \\
& +\left.q^{\delta_{\overline{i+1}, j}-\delta_{i, j}}\left(\frac{1-q_{2} \frac{q_{1}^{j} z}{w}}{1-\frac{q_{1}^{j} z}{w}}\right)^{\delta_{i, j}}\left(\frac{1-q_{3} \frac{q_{1}^{j} z}{w}}{1-q_{1}^{-1} \frac{q_{1}^{j} z}{w}}\right)^{\delta_{\overline{i+1}, j}} \Phi_{q_{1}}^{j}(w) E_{i}(z)\right|_{\mathcal{F}_{q_{1}, q_{3}}^{(1,0), k}(u)} .
\end{aligned}
$$


Using the Wick's theorem we normal order the vertex operators in the l.h.s. and r.h.s. of eq. (B.1):

$$
\begin{aligned}
& \left.E_{i}(z)\right|_{\mathcal{F}_{q_{1}, q_{3}}^{(1,0), k}(u)} \Phi_{q_{1}}^{j}(w)=z^{\delta_{i, j}-\delta_{\overline{i+1}, j}(-d)^{\delta} \delta_{\overline{i+1}, j}} \frac{\left(1-q_{2}^{-1} \frac{w}{q_{1}^{j} z}\right)^{\delta_{i, j}}}{\left(1-q_{1} \frac{w}{q_{1}^{j} z}\right)^{\delta_{\overline{i+1}, j}}}:\left.E_{i}(z)\right|_{\mathcal{F}_{q_{1}, q_{3}}^{(1,0), k}(u)} \Phi_{q_{1}}^{j}(w):, \\
& \left.\Phi_{q_{1}}^{j}(w) E_{i}(z)\right|_{\mathcal{F}_{q_{1}, q_{3}}^{(1,0), k}(u)}=\left(-q^{-1} q_{1}^{-j} w\right)^{\delta_{i, j}-\delta_{\overline{i+1}, j}} \frac{\left(1-\frac{q_{1}^{j} z}{w}\right)^{\delta_{i, j}}}{\left(1-q_{3} \frac{q_{1}^{j} z}{w}\right)^{\delta_{\overline{i+1}, j}}}:\left.E_{i}(z)\right|_{\mathcal{F}_{q_{1}, q_{3}}^{(1,0), k}(u)} \Phi_{q_{1}}^{j}(w): .
\end{aligned}
$$

Next we use the formal identity

$$
\frac{1}{1-x}=\delta(x)-\frac{x^{-1}}{1-x^{-1}},
$$

where $\delta(x)=\sum_{n \in \mathbb{Z}} x^{n}$ is the Dirac delta function. We apply eq. (B.4) to the rational prefactor in eq. (B.2). This gives

$$
\begin{aligned}
& \left.E_{i}(z)\right|_{\mathcal{F}_{q_{1}, q_{3}}^{(1,0), k}(u)} \Phi_{q_{1}}^{j}(w)=-q q_{1}^{j} w^{-1} \delta_{\overline{i+1}, j} \delta\left(\frac{w}{q_{1}^{j-1} z}\right):\left.E_{\overline{j-1}}\left(q_{1}^{1-j} w\right)\right|_{\mathcal{F}_{q_{1}, q_{3}}^{(1,0), k}(u)} \Phi_{q_{1}}^{j}(w): \\
& \quad+\left(q_{1}^{-j} q_{2}^{-1} w\right)^{\delta_{i, j}-\delta_{\overline{i+1}, j}}(-q)^{-\delta_{\overline{i+1}, j}} \frac{\left(1-q_{2} \frac{q_{1}^{j} z}{w}\right)^{\delta_{i, j}}}{\left(1-q_{1}^{-1} \frac{q_{1}^{j} z}{w}\right)^{\delta_{\overline{i+1}, j}}}:\left.E_{i}(z)\right|_{\mathcal{F}_{q_{1}, q_{3}}^{(1,0), k}(u)} \Phi_{q_{1}}^{j}(w): .
\end{aligned}
$$

The last remaining step in our check is to observe that

$$
:\left.E_{\overline{j-1}}\left(q_{1}^{1-j} w\right)\right|_{\mathcal{F}_{q_{1}, q_{3}}^{(1,0), k}(u)} \Phi_{q_{1}}^{j}(w):=q_{1}^{1-j} w \Phi_{q_{1}}^{\overline{j-1}}(w) .
$$

Substituting eq. (B.6) into eq. (B.5) and comparing with eq. (B.3) we find that eq. (B.1) indeed holds.

Open Access. This article is distributed under the terms of the Creative Commons Attribution License (CC-BY 4.0), which permits any use, distribution and reproduction in any medium, provided the original author(s) and source are credited.

\section{References}

[1] J.-t. Ding and K. Iohara, Generalization and deformation of Drinfeld quantum affine algebras, Lett. Math. Phys. 41 (1997) 181 [inSPIRE].

[2] K. Miki, $A(q, \gamma)$-analog of the $W_{1+\infty}$ algebra, J. Math. Phys. 48 (2007) 1.

[3] L.F. Alday, D. Gaiotto and Y. Tachikawa, Liouville Correlation Functions from Four-dimensional Gauge Theories, Lett. Math. Phys. 91 (2010) 167 [arXiv:0906.3219] [INSPIRE]. 
[4] N. Wyllard, $A(N-1)$ conformal Toda field theory correlation functions from conformal $N=2$ $\mathrm{SU}(N)$ quiver gauge theories, JHEP 11 (2009) 002 [arXiv:0907.2189] [INSPIRE].

[5] A. Mironov and A. Morozov, On AGT relation in the case of U(3), Nucl. Phys. B 825 (2010) 1 [arXiv:0908.2569] [INSPIRE].

[6] H. Nakajima, Instantons on ALE spaces, quiver varieties, and Kac-Moody algebras, Duke Math. J. 76 (1994) 365 [INSPIRE].

[7] I. Grojnowski, Instantons and affine algebras I: The Hilbert scheme and vertex operators, alg-geom/9506020 [inSPIRE].

[8] E. Carlsson, N. Nekrasov and A. Okounkov, Five dimensional gauge theories and vertex operators, Moscow Math. J. 14 (2014) 39 [arXiv: 1308.2465] [INSPIRE].

[9] Y. Zenkevich, Higgsed network calculus, JHEP 08 (2021) 149 [arXiv:1812.11961] [INSPIRE].

[10] H. Awata, B. Feigin and J. Shiraishi, Quantum Algebraic Approach to Refined Topological Vertex, JHEP 03 (2012) 041 [arXiv:1112 .6074] [INSPIRE].

[11] N.A. Nekrasov, Seiberg-Witten prepotential from instanton counting, Adv. Theor. Math. Phys. 7 (2003) 831 [hep-th/0206161] [INSPIRE].

[12] H. Awata, H. Kanno, A. Mironov, A. Morozov, K. Suetake and Y. Zenkevich, $(q, t)-K Z$ equations for quantum toroidal algebra and Nekrasov partition functions on ALE spaces, JHEP 03 (2018) 192 [arXiv: 1712.08016] [inSPIRE].

[13] J.-E. Bourgine and S. Jeong, New quantum toroidal algebras from $5 D \mathcal{N}=1$ instantons on orbifolds, JHEP 05 (2020) 127 [arXiv: 1906.01625] [INSPIRE].

[14] Y. Saito, Quantum toroidal algebras and their vertex representations, Publ. Res. Inst. Math. Sci. 34 (1998) 155 [q-alg/9611030].

[15] Y. Saito, K. Takemura and D. Uglov, Transform. Groups 3 (1998) 75 [q-alg/9702024].

[16] B. Feigin, M. Jimbo, T. Miwa and E. Mukhin, Branching rules for quantum toroidal gl ${ }_{n}$, Adv. Math. 300 (2016) 229 [arXiv: 1309.2147] [INSPIRE].

[17] A. Tsymbaliuk, Several realizations of Fock modules for quantum toroidal algebras of sl(n), Algebr. Represent. Theory 22 (2019) 177 [arXiv: 1603.08915] [INSPIRE].

[18] B. Feigin, M. Jimbo, T. Miwa and E. Mukhin, Representations of quantum toroidal $g_{n}$ J. Algebra 380 (2013) 78 [arXiv:1204.5378].

[19] B. Feigin, M. Jimbo and E. Mukhin, The $\left(\mathfrak{g l}_{m}, \mathfrak{g l}_{n}\right)$ Duality in the Quantum Toroidal Setting, Commun. Math. Phys. 367 (2019) 455 [arXiv: 1801.08433] [INSPIRE].

[20] I.B. Frenkel and V.G. Kac, Basic representations of affine Lie algebras and dual resonance models, Invent. Math. 62 (1980) 23.

[21] G. Segal, Unitarity Representations of Some Infinite Dimensional Groups, Commun. Math. Phys. 80 (1981) 301 [INSPIRE].

[22] K. Nagao, Quiver varieties and Frenkel-Kac construction, J. Algebra 321 (2009) 3764 [math/0703107].

[23] M. Wakimoto, Fock representations of the affine lie algebra A1(1), Commun. Math. Phys. 104 (1986) 605 [INSPIRE].

[24] L. Bezerra and E. Mukhin, Quantum toroidal algebra associated with $\mathfrak{g l}_{m \mid n}$, Algebr. Represent. Theory 24 (2021) 541 [arXiv: 1904. 07297] [INSPIRE]. 
[25] T. Procházka and M. Rapčák, Webs of W-algebras, JHEP 11 (2018) 109 [arXiv:1711.06888] [INSPIRE].

[26] T. Procházka and M. Rapčák, $\mathcal{W}$-algebra modules, free fields, and Gukov-Witten defects, JHEP 05 (2019) 159 [arXiv:1808.08837] [INSPIRE].

[27] B. Feigin and S. Gukov, Voa[m $\left[m_{4}\right]$, J. Math. Phys. 61 (2020) 012302 [arXiv:1806.02470] [INSPIRE].

[28] M. Rapčák, On extensions of $\mathfrak{g l} \widehat{(m \mid n)}$ Kac-Moody algebras and Calabi-Yau singularities, JHEP 01 (2020) 042 [arXiv: 1910.00031] [INSPIRE].

[29] J. Zhao, Orbifold Vortex and Super Liouville Theory, arXiv:1111.7095 [INSPIRE].

[30] T. Fujimori, T. Kimura, M. Nitta and K. Ohashi, Vortex counting from field theory, JHEP 06 (2012) 028 [arXiv: 1204.1968] [INSPIRE].

[31] Y. Yoshida, Localization of Vortex Partition Functions in $\mathcal{N}=(2,2)$ Super Yang-Mills theory, arXiv: 1101.0872 [INSPIRE].

[32] G. Bonelli, A. Tanzini and J. Zhao, Vertices, Vortices and Interacting Surface Operators, JHEP 06 (2012) 178 [arXiv:1102.0184] [INSPIRE]. 